\title{
Field theory for size- and charge asymmetric primitive model of ionic systems. Mean-field stability analysis and pretransitional effects
}

\author{
A.Ciach ${ }^{1}$, W.T. Góźdź ${ }^{1}$ and G.Stell ${ }^{2}$ \\ ${ }^{1}$ Institute of Physical Chemistry, Polish Academy of Sciences 01-224 Warszawa, Poland \\ ${ }^{2}$ Department of Chemistry, State University of \\ New York Stony Brook, NY 11 794-3400, USA
}

(Dated: July 4, 2018)

\begin{abstract}
The primitive model of ionic systems is investigated within a field-theoretic description for the whole range of size-,$\lambda$, and charge, $Z$, ratios of the two ionic species. Two order parameters (OP) are identified, and their relations to physically relevant quantities are described for various values of $\lambda$ and $Z$. Instabilities of the disordered phase associated with the two OP's are determined in the mean-field approximation (MF). In MF a gas-liquid separation occurs for any $Z$ and $\lambda \neq 1$. In addition, an instability with respect to various types of periodic ordering of the two kinds of ions is found. Depending on $\lambda$ and $Z$, one or the other transition is metastable in different thermdynamic states. The instabilities found in MF represent weak ordering of the majority of the instantaneous states, and are identified with structural loci associated with pretransitional effects.
\end{abstract}




\section{INTRODUCTION}

Universal features of collective phenomena can be determined within the framework of statistical mechanics with the interaction potentials approximated by highly simplified generic models. The generic model that allows for a prediction of general features of the collective phenomena in ionic systems as diverse as molten salts, electrolytes, room-temperature ionic liquids, as well as systems containing charged nanoparticles (including proteins), and/or charged colloidal particles, is the primitive model (PM). In the PM ions are represented by charged hard spheres, and the solvent (if present) is taken into account only through the dielectric constant [1, 2, 3]. In real systems the molecular structure of the solvent and multipole moments and polarizability of ions and solvent molecules affect the collective phenomena in the way that depends significantly on the specific properties of the ions and the solvent [4, 5], and is far from being understood. To identify and analyze specific effects in particular cases, however, one should know, in the first place, the phase diagram and structure in the generic case, where such effects are not present.

Despite the simplicity of the interaction potential in the PM, the dependence of the phase diagrams and the correlation functions on the charge,

$$
Z=\frac{e_{+}}{\left|e_{-}\right|}
$$

and the diameter,

$$
\lambda=\frac{\sigma_{+}}{\sigma_{-}}
$$

ratios of the two ionic species, respectively, is a largely unexplored problem. Until very

recently the theoretical studies concentrated mainly on the restricted primitive model (RPM) [1, 2, 3, 6], where $Z=\lambda=1$. In the RPM a gas-liquid separation occurs at low densities, and the bcc ionic crystal occurs at higher densities and sufficiently low temperatures. At still higher densities the fcc crystal with and without substitutional order at low and at high temperatures respectively is stable [3, 7].

Although much studied, the RPM is a quite artificial model, in the sense that no real ionic fluid has anions and cations of exactly the same size, although the size disparity is rather small in some, such as KCL. When one assumes full anion-cation symmetry, as in the RPM, there is a remarkable decoupling of the Ornstein-Zernike (OZ) integral equations that describe the density-density correlation function and the charge-charge correlation function, 
respectively. The two correlation functions are still indirectly coupled through the "closure relations" that must be added to the OZ equations to yield a closed set of equations, and the different approximations that have come into standard use in solving the primitive-model OZ equations- the mean spherical approximation, the hypernetted chain approximation, etc.are defined by different closure relations [1]. The total and the direct correlation function in the liquid theory are directly related to the (connected) correlation function and the vertex function respectively in the field theory (FT), and similar decoupling of the analogs of the OZ equations in the FT approach is found [3].

As soon as there is any degree of asymmetry, however, the density-density and chargecharge correlation functions are directly coupled, as are the OZ integral equations for these two functions [1], as well as their analogs in the FT [3, 8]. We shall not go through the details of this case here, and the interested reader will find those details in Section 2.2 of Ref.[1]. There is one aspect of the results there that is worth noting here explicitly, however. If there is only a very small degree of asymmetry- for example, if the effective diameters of the anions and cations are very nearly the same in a 1-1 electrolyte, one would expect that the RPM equations would be "almost" satisfied in some sense, despite the direct coupling one finds in the asymmetric case. This turns out to be the case- one finds that deviations from the RPM result only appear very close to the critical point for very small asymmetries. The smaller the deviations, the closer one must be to the critical point (cp) to see them. Recent theoretical [9, 10, 11, 12, 13, 14, 15, 16, 17] and simulation [18, 19, 20, 21, 22, 23, 24] results show that the PM with a small and moderate asymmetry exhibits qualitatively the same critical behavior as the RPM. In the asymmetric case the $\mathrm{cp}$ is shifted to lower temperatures and number densities (but higher volume fractions) [15, 16, 17, 18, 19, 20, 21, 22, 23, 24]. We expect to make further contact between our FT treatment for asymmetric ionic fluids and the general results of Ref.[1] concerning the critical behavior in future work. As far as we know, the effect of the size- and charge asymmetry on the gas-solid and liquid-solid transitions attracted much less attention so far.

Despite our general insight into the asymmetric case that has come out of the work of Ref.[1], and intensive studies of highly charged colloids [25, 26, 27, 28, 29, 30, 31, 32, 33, 34, 35], the case of the extreme asymmetry, $Z, \lambda \rightarrow \infty$, is not fully understood. This is because on the theoretical side the effect of approximations and assumptions on the results may be significant. In experiments [29, 30, 31, 34, 36] the interactions differ from the 
$\mathrm{PM}$, and the role of specific interactions and/or of the solvent properties is not known in full detail. Moreover, results may be sensitive to impurities, nonequilibrium effects etc.. Last but not least, it is very difficult to obtain reliable simulation results for the phase diagram when $Z, \lambda \rightarrow \infty$. To ensure charge neutrality $Z$ anions must be present per each cation, and in studies of collective phenomena the number of cations $N_{+}$must be large. Typical experiments correspond to $Z \sim 10^{4}$, i.e. to $\sim 10^{4} N_{+}$ions in the simulation box. Because of the above reasons, there is no consensus concerning the phase behavior in the PM in the case of the extreme asymmetry, and the results of some experiments [30, 34] are considered as controversial [33, 35]. The controversy concerns mainly the occurrence of the gas-liquid separation at room temperature in the case of monovalent counterions. Simulations indicate that at room temperatures the gas-liquid separation for monovalent counterions does not occur, but occurs for divalent counterions [33]. Preliminary fieldtheoretic results [8] indicate that when the size and the charge of the cation are several orders of magnitude larger than the size and the charge of the anion, the gas-liquid type phase separation is preempted by a transition between gas and a colloidal crystal for a large range of temperatures, including room temperature [8]. The predictions of Ref. [8] are limited to the mean field (MF) approximation, and the effect of fluctuations on the transition lines remains to be determined along the same lines as in the case of the RPM [37]. The phase behavior for higher volume fractions is also not quite clear. Many studies suggest a transition between the bcc and fcc crystals, and next a gel or glass formation when the volume fraction of colloidal particles increases [36, 38, 39]. Additional counterions and coions significantly influence the phase behavior [6, 27, 28, 36, 39, 40]. Note that the above phase behavior is quite different than the behavior in the RPM.

The way the global phase diagram evolves when the asymmetry parameters are varied has not been systematically investigated yet. The values of $Z$ and $\lambda$ have a strong effect on formation of ordered periodic structures of a crystal type. Coulomb interactions support structures in which the positive charges are compensated by the negative charges in regions as small as possible. On the other hand, precise compensation of charges in small regions may lead to an increase of entropy. The electrostatic and the entropic contributions to the grand potential both depend on the geometrical constraints. These constraints are due to a packing of $Z$ spheres of a diameter $\sigma_{-}$and one sphere of a diameter $\sigma_{+}=\lambda \sigma_{-}$into a charge-neutral aggregate, and a possibility of periodically repeating this aggregate in space. 
Such an ordered structure is favored compared to the disordered phase when the entropic contribution is sufficiently small compared to the electrostatic energy. Combination of the geometric constrains and the charge-neutrality condition for some values of $Z$ and $\lambda$ may promote ordering, while in other cases may suppress ordering. The competition between the periodic ordering and the phase separation also depends on $Z$ and $\lambda$. Thus, rich and complex phase behavior in the parameter space $\left(\zeta, T^{*} ; Z, \lambda\right)$ may be expected, where $\zeta$ and $T^{*}$ are the volume fraction of ions and temperature in the standard reduced (dimensionless) units [1, 3], defined in the next section. Depending on the values of $Z$ and $\lambda$, phase equilibria on the $\left(\zeta, T^{*}\right)$ phase diagram may include some (or all) of the following transitions: gas-crystal, gas-liquid, liquid-crystal, crystal-glass, crystal-gel or transition between different crystalline phases. For different $Z$ and $\lambda$ the above phase equilibria may occur in quite different parts of the $\left(\zeta, T^{*}\right)$ phase diagram.

Some insight into the dependence of the phase behavior on $Z$ and $\lambda$ can be gained from studies of pretransitional effects in the disordered phase. Such effects may be divided into two categories: effects associated with the gas-liquid separation and effects associated with the crystallization. Theoretical studies [1, 3, 15, 16, 41] emphasize the role of cluster formation for the gas-liquid separation in ionic systems. Recent Monte Carlo (MC) 19, 20, 21, 22, 23, 24, 42, 43, 44, 45], molecular dynamics (MD) [46] and Brownian dynamics (BD) [47] simulation studies provide interesting information on clustering in the size- and charge asymmetric PM. In Refs. [42, 43, 46, 47] the simulations were performed at room temperature for several values of the volume fraction of the larger ions, for several sizes of both ionic species (size ratio 5-10), and for several values of the charge on the larger ion ( 10-20 elementary charges). The cluster formation was studied for mono- di- and trivalent counterions for fixed values of the remaining parameters. In equilibrium the instantaneous structures are much more uniform in the case of monovalent counterions than in the other cases, where 'living' clusters containing two or more large ions are formed [43, 47]. For trivalent counterions a substantial fraction of the clusters was found to be neutral. Attractions between the neutral clusters are similar to the attractions between molecules, and may lead to the phase separation. The dependence on valency of counterions for a fixed charge on the larger ion and for fixed temperature is equivalent to a dependence on $Z$ and $T^{*}$. The question of the region in the parameter space $\left(\zeta, T^{*} ; Z, \lambda\right)$ corresponding to a formation of neutral clusters remains open. 
Pretransitional effects associated with weak long-range ordering are a subject of the present study. By analogy with the short-range ordering of the instantaneous states described above, we expect that such effects include long-range ordering of the instantaneous states. In the long-range ordered instantaneous states a local deviation from the uniform distribution of ions is periodically repeated in space.

In this work we present an overview of the collective phenomena in the PM for the whole range of $Z$ and $\lambda$. Our FT analysis allows us to identify the dominant deviations from the uniform distribution of ions. We find structural lines that separate the stability region of the disordered phase on the $\left(\zeta, T^{*}\right)$ phase diagram in the high-temperature part where the homogeneous instantaneous states are more probable than periodic states with small amplitude, and in the low- $T^{*}$ part, where the majority of the instantaneous states exhibit weak long-range order. At the structural line the amplitude of the periodic deviation from the uniform distribution of ions in the most probable instantaneous states increases continuously from zero. We stress that the structural line is neither a phase transition in the thermodynamic sense, nor a true spinodal. Determination of the probability of the periodic instantaneous states with large amplitudes, as well as determination of the phase transitions requires further studies, for which our analysis should serve as a starting point.

In sec.II we describe the field theory for the PM. We introduce the concept of the structural line and show that this line coincides with the MF approximation for the spinodal line. In sec.III general expressions for the eigenmodes and boundary of stability of the uniform phase in MF (structural lines) are given. The nature of the eigenmodes is discussed, and the dominant instantaneous states are identified in the same section. Sec.IV is devoted to the case of equal sizes. MF-spinodal (structural) lines associated with the gas-liquid separation are described in detail in sec.V, and in sec.VI the structural lines associated with periodic ordering are discussed. Explicit results for representative values of $Z$ and $\lambda$ are also presented in these two sections. A short summary is given in sec.VII. 


\section{FIELD-THEORETIC DESCRIPTION OF THE PRIMITIVE MODEL}

\section{A. Coarse graining of the PM}

We consider a mixture of positively charged ions of a charge $e_{+}$and diameter $\sigma_{+}$, and negatively charged ions of a charge $e_{-}=-\left|e_{-}\right|$and diameter $\sigma_{-}$in a structureless, incompressible solvent. In the PM the interaction potential of a pair $\alpha, \beta$, where the Greek indices denote + or - , is infinite for distances smaller than $\sigma_{\alpha}+\sigma_{\beta}$, i.e. we assume hard-core repulsions. The electrostatic potentials $V_{\alpha \beta}\left(\mathbf{r}_{1}-\mathbf{r}_{2}\right)$ between different pairs of ions $\alpha, \beta$ are the following

$$
V_{\alpha \beta}(r)=\frac{e_{\alpha} e_{\beta}}{D r} \theta\left(r-\sigma_{\alpha \beta}\right)
$$

where

$$
\sigma_{\alpha \beta}=\frac{1}{2}\left(\sigma_{\alpha}+\sigma_{\beta}\right)
$$

and $D$ is the dielectric constant of the solvent. The $\theta$-functions above prevent from the contributions to the electrostatic energy coming from overlapping hard spheres, i.e. we do not include the electrostatic self-energy.

In the field-theoretic approach we consider local densities of the ionic species, $\rho_{+}(\mathbf{r})$ and $\rho_{-}(\mathbf{r})$, and we include only smooth functions. For particular fields $\rho_{+}(\mathbf{r}), \rho_{-}(\mathbf{r})$ we assume that the electrostatic energy assumes the form

$$
U\left[\rho_{+}, \rho_{-}\right]=\frac{1}{2} \int d \mathbf{r}_{1} \int d \mathbf{r}_{2} \rho_{\alpha}\left(\mathbf{r}_{1}\right) V_{\alpha \beta}\left(\mathbf{r}_{1}-\mathbf{r}_{2}\right) \rho_{\beta}\left(\mathbf{r}_{2}\right)
$$

where summation convention for the Greek indices is used here and below. The probability that the local densities assume a particular form $\rho_{+}(\mathbf{r}), \rho_{-}(\mathbf{r})$ is proportional to $\exp \left[-\beta\left(U\left[\rho_{+}, \rho_{-}\right]-\mu_{+} N_{+}-\mu_{-} N_{-}\right)\right]$, where $N_{+}=\int d \mathbf{r} \rho_{+}(\mathbf{r})$ and $N_{-}=\int d \mathbf{r} \rho_{-}(\mathbf{r})$ are the number of positive charged and negative charged ions respectively. The chemical potentials of the two ionic species, $\mu_{+}$and $\mu_{-}$, are not independent, and have to be consistent with the requirement of charge neutrality,

$$
\int d \mathbf{r} \rho_{+}(\mathbf{r}) e_{+}=\int d \mathbf{r} \rho_{-}(\mathbf{r})\left|e_{-}\right|
$$

Whereas the charge neutrality condition must be obeyed in macroscopic regions, it can be violated locally, in regions containing a small number of ions. The probability that the local densities assume the form $\rho_{+}(\mathbf{r}), \rho_{-}(\mathbf{r})$ is also proportional to the number of all microscopic 
states compatible with $\rho_{+}(\mathbf{r}), \rho_{-}(\mathbf{r})$. The number of all states can be written in the form $\exp (\beta T S)$, where $\beta=(k T)^{-1}$, and where $S, T$ and $k$ are the entropy, temperature and the Boltzmann constant respectively. From the above it follows that the Boltzmann factor assumes the form

$$
p\left[\rho_{+}, \rho_{-}\right]=\Xi^{-1} \exp \left(-\beta \Omega^{M F}\left[\rho_{+}, \rho_{-}\right]\right)
$$

where

$$
\Xi=\int D \rho_{+} \int D \rho_{-} \exp \left(-\beta \Omega^{M F}\left[\rho_{+}, \rho_{-}\right]\right)
$$

and

$$
\Omega^{M F}\left[\rho_{+}, \rho_{-}\right]=F_{h}\left[\rho_{+}, \rho_{-}\right]+U\left[\rho_{+}, \rho_{-}\right]-\mu_{+} \int d \mathbf{r} \rho_{+}(\mathbf{r})-\mu_{-} \int d \mathbf{r} \rho_{-}(\mathbf{r})
$$

is the grand potential in the system where the local concentrations of the two ionic species are constrained to have the forms $\rho_{+}(\mathbf{r}), \rho_{-}(\mathbf{r})$. In Eq.(9) $F_{h}=-T S$ is the Helmholtz free energy of the hard-core reference system. In the local density approximation $F_{h}\left[\rho_{+}, \rho_{-}\right]=$ $\int d \mathbf{r} f_{h}\left(\rho_{+}(\mathbf{r}), \rho_{-}(\mathbf{r})\right)$, and $f_{h}$ consists of the ideal-gas contribution plus the excess free-energy density of hard-spheres with different diameters $f_{h}^{e x}$,

$$
\beta f_{h}\left(\rho_{+}(\mathbf{r}), \rho_{-}(\mathbf{r})\right)=\sum_{\alpha} \rho_{\alpha}(\mathbf{r})\left(\ln \left(\Lambda_{\alpha}^{3} \rho_{\alpha}(\mathbf{r})\right)-1\right)+\beta f_{h}^{e x}\left(\rho_{+}(\mathbf{r}), \rho_{-}(\mathbf{r})\right) .
$$

The $\Lambda_{\alpha}$ is the thermal de Broglie wavelength of species $\alpha, \alpha=+,-$. Here for $f_{h}^{e x}$ we assume the Percus-Yevick compressibility route to the Helmholtz free-energy of a hardsphere mixture with size asymmetry [48]. The above theory is equivalent to an approximate version [49] of the exact collective-variables theory [50, 51].

As a length unit we choose $\sigma_{+-}$. The dimensionless densities and inverse temperature are chosen as

$$
\rho_{+}^{*}=\rho_{+} \sigma_{+-}^{3}, \quad \rho_{-}^{*}=\rho_{-} \sigma_{+-}^{3}, \quad \beta^{*}=\beta \frac{e_{+}\left|e_{-}\right|}{D \sigma_{+-}} .
$$

As thermodynamic variables we choose $T^{*}=1 / \beta^{*}$ and the volume fraction of all ions,

$$
\zeta=\frac{\pi}{6}\left(\rho_{0+} \sigma_{+}^{3}+\rho_{0-} \sigma_{-}^{3}\right)
$$

The volume fraction of all ions is related to the quantity proportional to the number density of all ionic species,

$$
s=\frac{\pi}{6}\left(\rho_{0+}^{*}+\rho_{0-}^{*}\right)=\sigma_{+-}^{3}\left(\bar{N}_{+}+\bar{N}_{-}\right) / V
$$


where $V$ is the volume and $\bar{N}_{ \pm}$denote the average numbers of positive and negative charges for given chemical potentials. The relation between $\zeta$ and $s$ is given by

$$
\zeta=s\left[1+3 \delta(\delta-\nu)-\delta^{3} \nu\right]
$$

We also define the parameters describing the charge- and size asymmetry,

$$
\nu=\frac{Z-1}{Z+1}
$$

and

$$
\delta=\frac{\lambda-1}{\lambda+1}
$$

For $\delta \nu>0(\delta \nu<0)$ the charge at the larger ion is larger (smaller). Because of the symmetry, we limit ourselves to $\delta>0$ and consider $-1 \leq \nu \leq 1$.

Let us focus on homogeneous instantaneous states corresponding to the extremum of $\Omega^{M F}$. The densities in the uniform states, $\rho_{\alpha}^{*}(\mathbf{r})=$ const., corresponding to $\delta \Omega^{M F} / \delta \rho_{\alpha}^{*}=0$, will be denoted by $\rho_{0 \alpha}^{*}$. At sufficiently high $T^{*}$, homogeneous instantaneous states correspond to the global maximum of the Boltzmann factor (7), i.e. to the global minimum of $\Omega^{M F}$. At low temperatures the extremum of $\Omega^{M F}$ may correspond to either a local minimum, a saddle point or to a maximum. For small deviations of $\rho_{+}^{*}(\mathbf{x})$ and $\rho_{-}^{*}(\mathbf{x})$ from $\rho_{0+}^{*}$ and $\rho_{0-}^{*}$ respectively, the functional (91) can be expanded. The expansion can be truncated for $\rho_{\alpha}^{*}-\rho_{0 \alpha}^{*} \rightarrow 0$. We write $\Omega^{M F}$ in the form

$$
\Omega^{M F}=\Omega_{0}+\Omega_{2}+\Omega_{\text {int }},
$$

where $\Omega_{0}=\Omega^{M F}\left[\rho_{0+}^{*}, \rho_{0-}^{*}\right]$ is the value of the functional at the extremum, and $\Omega_{2}$ denotes the Gaussian part of the functional. In Fourier representation we have

$$
\beta \Omega_{2}\left[\Delta \tilde{\rho}_{+}(\mathbf{k}), \Delta \tilde{\rho}_{-}(\mathbf{k})\right]=\frac{1}{2} \int \frac{d \mathbf{k}}{(2 \pi)^{3}} \Delta \tilde{\rho}_{\alpha}(-\mathbf{k}) \tilde{C}_{\alpha \beta}^{0}(\mathbf{k}) \Delta \tilde{\rho}_{\beta}(\mathbf{k}),
$$

where $\Delta \tilde{\rho}_{\alpha}(\mathbf{k})$ is the Fourier transform of $\Delta \rho_{\alpha}^{*}(\mathbf{r})=\rho_{\alpha}^{*}(\mathbf{r})-\rho_{0 \alpha}^{*}$. In Eq.(18) and hereafter the wave numbers are in $\sigma_{+-}^{-1}$ units. The second functional derivative of $\Omega^{M F}\left[\rho_{+}^{*}, \rho_{-}^{*}\right]$ at $\rho_{\alpha}^{*}=\rho_{0 \alpha}^{*}$ consists of two terms,

$$
\tilde{C}_{\alpha \beta}^{0}(\mathbf{k})=a_{\alpha \beta}+\beta \tilde{V}_{\alpha \beta}(k)
$$

The first term is given by

$$
a_{\alpha \beta}=\frac{\partial \beta f_{h}}{\partial \rho_{\alpha}^{*} \partial \rho_{\beta}^{*}},
$$


where the derivative is taken at $\rho_{\alpha}^{*}=\rho_{0 \alpha}^{*}$. Explicit expressions for $a_{\alpha \beta}$ are given in Appendix A. We have used the chemical potentials for the asymmetric hard-sphere mixture obtained in the Percus-Yevick approximation in Ref.[48]. The second term in $\tilde{C}_{\alpha \beta}^{0}(\mathbf{k})$ is the Fourier transform of the potential (3) and we find

$$
\begin{gathered}
\beta \tilde{V}_{++}(k)=\beta^{*} Z \frac{4 \pi \cos \left(k r_{+}\right)}{k^{2}} \\
\beta \tilde{V}_{--}(k)=\beta^{*} Z^{-1} \frac{4 \pi \cos \left(k r_{-}\right)}{k^{2}} \\
\beta \tilde{V}_{+-}(k)=-\beta^{*} \frac{4 \pi \cos k}{k^{2}}
\end{gathered}
$$

where

$$
r_{ \pm}=\frac{\sigma_{ \pm}}{\sigma_{+-}}=1 \pm \delta
$$

The higher-order part of the functional (17) can be written in the form

$$
\beta \Omega_{\text {int }}=\int d \mathbf{r}\left[\frac{a_{\alpha \beta \gamma}}{3 !} \Delta \rho_{\alpha}(\mathbf{r}) \Delta \rho_{\beta}(\mathbf{r}) \Delta \rho_{\gamma}(\mathbf{r})+\frac{a_{\alpha \beta \gamma \nu}}{4 !} \Delta \rho_{\alpha}(\mathbf{r}) \Delta \rho_{\beta}(\mathbf{r}) \Delta \rho_{\gamma}(\mathbf{r}) \Delta \rho_{\nu}(\mathbf{r})\right]+\ldots
$$

where

$$
a_{\alpha \beta \gamma}=\frac{\partial a_{\alpha \beta}}{\partial \rho_{\gamma}^{*}} \quad \text { and } \quad a_{\alpha \beta \gamma \nu}=\frac{\partial a_{\alpha \beta \gamma}}{\partial \rho_{\nu}^{*}}
$$

and the derivatives are taken at $\rho_{\alpha}^{*}=\rho_{0 \alpha}^{*}$.

The RPM limit of the above general model corresponds to $\delta=\nu=0$ and was studied before in Ref.[3, 52]. The opposite, colloid limit of $\delta \rightarrow 1, \nu \rightarrow 1(\lambda, Z \rightarrow \infty)$ with $s=O\left(Z^{0}\right)$ (i.e. $\left.\zeta=O\left(Z^{-1}\right)\right)$ is described in Ref.[8].

\section{B. Correlation and vertex functions and their generating functionals}

In the coarse-grained description the densities $\rho_{\alpha}^{*}(\mathbf{r})$ play the role of the microstates. Thus, the standard definitions of thermodynamic potentials and correlations functions can be applied. In particular, the grand-thermodynamic potential in a presence of external fields $J_{+}(\mathbf{r})$ and $J_{-}(\mathbf{r})$ is given by

$$
\Omega\left[J_{+}(\mathbf{r}), J_{-}(\mathbf{r})\right]=-k T \log \Xi\left[J_{+}(\mathbf{r}), J_{-}(\mathbf{r})\right] .
$$

where

$$
\Xi\left[J_{+}(\mathbf{r}), J_{-}(\mathbf{r})\right]=\int D \rho_{+} \int D \rho_{-} \exp \left[-\beta\left(\Omega^{M F}\left[\rho_{+}, \rho_{-}\right]-\int_{\mathbf{r}} J_{\alpha}(\mathbf{r}) \rho_{\alpha}(\mathbf{r})\right)\right]
$$


The external fields may play an important role in some experimental cases. For systems where no external fields are present, as in our case, they are introduced as auxiliary fields for computational reasons. The Legeandre transform

$$
F\left[\bar{\rho}_{+}(\mathbf{r}), \bar{\rho}_{-}(\mathbf{r})\right]=\Omega\left[J_{+}(\mathbf{r}), J_{-}(\mathbf{r})\right]+\int_{\mathbf{r}} J_{\alpha}(\mathbf{r}) \bar{\rho}_{\alpha}(\mathbf{r}),
$$

where $\bar{\rho}_{\alpha}=-\delta \Omega\left[J_{+}(\mathbf{r}), J_{-}(\mathbf{r})\right] / \delta J_{\alpha}$, is the density functional. The $-\Omega\left[J_{+}(\mathbf{r}), J_{-}(\mathbf{r})\right]$ and $F\left[\bar{\rho}_{+}(\mathbf{r}), \bar{\rho}_{-}(\mathbf{r})\right]$ are the generating functionals for the correlation and the vertex functions respectively [53, 54]. The two-point vertex function $C_{\alpha \beta}=\delta^{2} F / \delta \bar{\rho}_{\alpha} \delta \bar{\rho}_{\beta}$ is related to the analog of the direct correlation function $c_{\alpha \beta}$. In Fourier representation this relation has the form [53]

$$
\tilde{C}_{\alpha \beta}(k)=\frac{\delta_{\alpha \beta}^{K r}}{\left\langle\rho_{\alpha}\right\rangle}-\tilde{c}_{\alpha \beta}(k),
$$

where $\delta_{\alpha \beta}^{K r}$ is the Kronecker symbol, and $\left\langle\rho_{\alpha}\right\rangle$ is the average density of the specie $\alpha$. In terms of the vertex functions the FT analogs of the $\mathrm{OZ}$ equations take the simple form

$$
\tilde{C}_{\alpha \beta}(k) \tilde{G}_{\beta \gamma}(k)=\delta_{\alpha \gamma}^{K r},
$$

where the Fourier transform $\tilde{G}_{\alpha \beta}(k)$ of the two-point correlation function,

$$
G_{\alpha \beta}(r)=\left\langle\Delta \rho_{\alpha}(\mathbf{0}) \Delta \rho_{\beta}(\mathbf{r})\right\rangle=-\frac{\delta^{2} \Omega}{\delta J_{\alpha}(\mathbf{0}) \delta J_{\beta}(\mathbf{r})},
$$

is related to the analog of the total correlation function $h_{\alpha \beta}$ by [53]

$$
\tilde{G}_{\alpha \beta}(k)=\delta_{\alpha \beta}^{K r}\left\langle\rho_{\alpha}\right\rangle+\tilde{h}_{\alpha \beta}(k)\left\langle\rho_{\alpha}\right\rangle\left\langle\rho_{\beta}\right\rangle .
$$

The FT approach with the local-density approximation (10) is designed for a description of the long range ordering that determines phase transitions. Due to the coarse-graining (or 'smearing' of the hard-spheres structure) the correlation functions for distances $<\sigma_{+}+\sigma_{-}$ are not correctly reproduced.

\section{Mean-field approximation}

In practice the exact forms of $\Omega$ and $F$ cannot be obtained. In order to adopt standard approximations to the PM, let us focus on the most probable instantaneous distributions of ions $\rho_{0 \alpha}^{*}(\mathbf{r})$, corresponding to the global maximum of the Boltzmann factor (in the presence of the external fields the latter is proportional to the integrand in Eq.(28)). At low $T^{*}$ 
the most probable distributions may not be uniform; in particular, crystalline phases are characterized by distributions of ions that are periodic in space. We rewrite Eqs.(27) and (29) in the form

$$
\Omega\left[J_{+}, J_{-}\right]=\Omega^{M F}\left[\rho_{0+}^{*}(\mathbf{r}), \rho_{0-}^{*}(\mathbf{r})\right]-\int_{\mathbf{r}} J_{\alpha}(\mathbf{r}) \rho_{0 \alpha}^{*}(\mathbf{r})-k T \log \Delta \Xi\left[J_{+}, J_{-}\right],
$$

and

$$
F\left[\bar{\rho}_{+}, \bar{\rho}_{-}\right]=\Omega^{M F}\left[\rho_{0+}^{*}(\mathbf{r}), \rho_{0-}^{*}(\mathbf{r})\right]+\int_{\mathbf{r}} J_{\alpha}(\mathbf{r})\left(\bar{\rho}_{\alpha}(\mathbf{r})-\rho_{0 \alpha}^{*}(\mathbf{r})\right)-k T \log \Delta \Xi\left[J_{+}, J_{-}\right]
$$

where

$$
\Delta \Xi\left[J_{+}, J_{-}\right]=\int D \Delta \rho_{+} \int D \Delta \rho_{-} \exp \left[-\beta\left(\Delta \Omega^{M F}\left[\Delta \rho_{+}, \Delta \rho_{-}\right]-\int_{\mathbf{r}} J_{\alpha}(\mathbf{r}) \Delta \rho_{\alpha}(\mathbf{r})\right)\right],
$$

and

$$
\Delta \Omega^{M F}\left[\Delta \rho_{+}, \Delta \rho_{-}\right]=\Omega^{M F}\left[\rho_{0+}^{*}+\Delta \rho_{+}, \rho_{0-}^{*}+\Delta \rho_{-}\right]-\Omega^{M F}\left[\rho_{0+}^{*}, \rho_{0-}^{*}\right]
$$

The last term in Eq.(34) is the contribution to the grand potential associated with fluctuations $\Delta \rho_{+}, \Delta \rho_{-}$around the most probable distributions, $\rho_{0+}^{*}(\mathbf{r})$ and $\rho_{0-}^{*}(\mathbf{r})$. In the $\mathrm{MF}$ approximation this term is just neglected, and the grand thermodynamic potential is approximated by the minimum of $\Omega^{M F}\left[\rho_{+}, \rho_{-}\right]-\int_{\mathbf{r}} J_{\alpha}(\mathbf{r}) \rho_{\alpha}(\mathbf{r})$ with respect to $\rho_{\alpha}$ for fixed $J_{\alpha}(\mathbf{r})$. The average values of the local densities,

$$
\begin{array}{r}
\left\langle\rho_{\alpha}(\mathbf{r})\right\rangle=\bar{\rho}_{\alpha}(\mathbf{r})=\rho_{0 \alpha}^{*}(\mathbf{r})+\Delta \Xi\left[J_{+}, J_{-}\right]^{-1} \int D \Delta \rho_{+} \int D \Delta \rho_{-} \Delta \rho_{\alpha}(\mathbf{r}) \times \\
\exp \left[-\beta\left(\Delta \Omega^{M F}\left[\Delta \rho_{+}, \Delta \rho_{-}\right]-\int_{\mathbf{r}} J_{\alpha}(\mathbf{r}) \Delta \rho_{\alpha}(\mathbf{r})\right)\right],
\end{array}
$$

in MF are identified with their most probable values $\rho_{0 \alpha}^{*}(\mathbf{r})$, i.e. the fluctuation contribution in Eq.(38) is neglected. Beyond $\operatorname{MF}\left\langle\rho_{\alpha}(\mathbf{r})\right\rangle$ and $\rho_{0 \alpha}^{*}(\mathbf{r})$ may differ from each other, as found in the case of the RPM [37, 55]. In the context of the PM the role of the fluctuation contribution in Eqs.(34) and (38) will be studied in the future work, by following the Brazovskii approach [56] applied to the RPM in Ref. [41].

In the MF approximation the generating functionals for the vertex and the correlation functions, $F\left[\rho_{+}, \rho_{-}\right]$and $-\Omega\left[J_{+}, J_{-}\right]$, reduce to $\Omega^{M F}\left[\rho_{+}, \rho_{-}\right]$and $-\left(\Omega^{M F}\left[\rho_{+}, \rho_{-}\right]-\right.$ $\left.\int_{\mathbf{r}} J_{\alpha}(\mathbf{r}) \rho_{\alpha}(\mathbf{r})\right)$ respectively. For $J_{\alpha}=0$ the two-point correlation and vertex functions, $G_{\alpha \beta}^{0}$ and $C_{\alpha \beta}^{0}$ respectively, in the above version of MF are identical to the corresponding functions in the Gaussian approximation. In the Gaussian approximation $\Omega^{M F}$ in the Boltzmann factor (17) is approximated by Eq.(17) with the last term neglected. For $\Omega^{M F}=\Omega_{2}$ 
the exact two-point correlation functions, $G_{\alpha \beta}^{0}$, are related to $C_{\alpha \beta}^{0}$ given in Eq.(19) by the OZ equations analogous to Eq.(31).

\section{Phase transitions, spinodals and pretransitional effects}

Let us focus on the FT predictions for the phase diagram in the case of $J_{\alpha}=0$. The stable (metastable) phases correspond to the global (local) minimum of $F\left[\bar{\rho}_{+}, \bar{\rho}_{-}\right]$(Eqs.(29) and (35)). Two minima corresponding to different forms of $\bar{\rho}_{\alpha}(\mathbf{r})$ are of equal depth at the coexistence between the corresponding phases. Let us assume that the global minimum of $F\left[\bar{\rho}_{+}, \bar{\rho}_{-}\right]$corresponds to $\rho_{0 \alpha}^{*}(\mathbf{r})$, which deviates significantly from the constant function $\rho_{0 \alpha}^{*}$, corresponding to another extremum of $F\left[\bar{\rho}_{+}, \bar{\rho}_{-}\right]$. Crystalline phases are characterized by such density distributions. The minmum of $F$ corresponding to such nonuniform distributions can be found only when $\Omega_{\text {int }}$ in Eqs.(17) and (35) is included, because the Gaussian part $\Omega_{2}$ describes correctly only infinitesimal deviations from the uniform distributions of ions $\rho_{0 \alpha}^{*}(\mathbf{r}) . \Omega_{\text {int }}$ is given in terms of the three- and higher-order vertex functions in the MF approximation. Thus, the two-point vertex (or correlation) functions are not sufficient to find the transitions to the ordered phases; it is essential to include in Eq. (35) the higher-order vertex functions as well.

Let us consider the boundary of the global (local) stability of the uniform phase. The extremum of $F$ changes character at the corresponding continuous-transition (spinodal) line. At the high and at the low- $T^{*}$ side of this line the homogeneous phase corresponds to a global (local) minimum, and to a saddle point of $F$ respectively. More precisely, for given $\zeta$ the boundary of stability of the uniform phase is given by the highest $T^{*}$, for which $\operatorname{det} \tilde{C}_{\alpha \beta}(k)=0\left(\delta F / \delta \bar{\rho}_{\alpha} \delta \bar{\rho}_{\beta}\right.$ is not positive definite) for some $k=k_{b}$. In MF the boundary of the stability of the uniform phase is obtained with $\tilde{C}_{\alpha \beta}(k)$ approximated by $\tilde{C}_{\alpha \beta}^{0}(k)$ (Eq.(19)). From the earlier studies [41, 56] it follows that when $\operatorname{det} \tilde{C}_{\alpha \beta}^{0}(k)=0$ for $k \neq 0$, the fluctuation contribution to $F$ in Eq. (35) is comparable to $\Omega^{M F}$. Hence, the difference between $\tilde{C}_{\alpha \beta}(k)$ and $\tilde{C}_{\alpha \beta}^{0}(k)$ may be comparable to $\tilde{C}_{\alpha \beta}^{0}(k)$. In such a case the fluctuation contributions to Eqs. (35)-(38) lead to significant shifts of the phase boundaries. In addition, if a continuous transition to a phase with periodic densities is found in MF, a fluctuation-induced firstorder transition is expected at significantly lower temperatures [41, 56] when the fluctuation contribution is included. 
The analysis of the stability of $\Omega^{M F}\left[\rho_{+}, \rho_{-}\right]$cannot give information on the actual instability of the disordered phase, but may give information on the pretransitional effects associated with weak long-range ordering of the instantaneous states. Let us compare the probability that an instantaneous state $\Delta \rho_{+}(\mathbf{x}) \neq 0, \Delta \rho_{-}(\mathbf{x}) \neq 0$ occurs, with the probability of finding the homogeneous instantaneous state $\Delta \rho_{+}=\Delta \rho_{-}=0$. According to Eqs.(7) and (17), the probability ratio is

$$
\frac{p\left[\Delta \rho_{+}, \Delta \rho_{-}\right]}{p[0,0]}=e^{-\beta\left(\Omega_{2}+\Omega_{\text {int }}\right)} .
$$

Let the smaller eigenvalue of $\tilde{C}_{\alpha \beta}^{0}(k)$ in Eq.(18) be denoted by $\tilde{C}_{\phi \phi}^{0}(k)$. The eigenmode $\tilde{\phi}(\mathbf{k})$ corresponding to $k=k_{0}$ can be written as a linear combination of the functions

$$
\tilde{g}_{i}\left(\mathbf{k} \mid k_{0}\right)=\frac{(2 \pi)^{d}}{\sqrt{2}}\left(w \delta\left(\mathbf{k}-\mathbf{k}_{0 i}\right)+w^{*} \delta\left(\mathbf{k}+\mathbf{k}_{0 i}\right)\right)
$$

with different directions of the vectors $\mathbf{k}_{0 i}$, where $\left|\mathbf{k}_{0 i}\right|=k_{0}$ and $w w^{*}=1$. Let us focus on the instantaneous structure that has the form $\tilde{\phi}(\mathbf{k})=\Phi_{i} \tilde{g}_{i}\left(\mathbf{k} \mid k_{0}\right)$, and the amplitude of the other eigenmode of $\tilde{C}_{\alpha \beta}^{0}\left(k_{0}\right), \tilde{\eta}(\mathbf{k})$, vanishes. From Eq. (18) we have $\beta \Omega_{2}=V \sum_{i} \Phi_{i}^{2} \tilde{C}_{\phi \phi}\left(k_{0}\right) / 2$, where $V$ is the volume of the system. Then Eq.(39) takes the form

$$
\frac{\bar{p}\left[\Phi_{i} \tilde{g}_{i}\left(\mathbf{k} \mid k_{0}\right), 0\right]}{p[0,0]}=\exp \left[-\frac{\sum_{i} \Phi_{i}^{2} V}{2}\left(\tilde{C}_{\phi \phi}\left(k_{0}\right)+O\left(\Phi_{j}\right)\right)\right] .
$$

In the above $\bar{p}$ is the probability (39) as a function of the new variables, $\tilde{\phi}(\mathbf{k})$ and $\tilde{\eta}(\mathbf{k})$.

The sign of the term $O\left(\Phi_{j}\right)$ depends on the form of $\Omega_{\text {int }}$ in Eq.(17), and on the form of $\tilde{\phi}(\mathbf{k})$. Here we limit ourselves to infinitesimal amplitudes $\Phi_{i}$. When the term $O\left(\Phi_{i}\right)$ in (41) is negligible, the probability ratio is larger or smaller from unity for $\tilde{C}_{\phi \phi}\left(k_{0}\right)<0$ or for $\tilde{C}_{\phi \phi}\left(k_{0}\right)>0$ respectively. For given $\zeta$, the solution of $\tilde{C}_{\phi \phi}\left(k_{0}\right)=0$ yields the temperature $T^{*}\left(\zeta ; k_{0}\right)$, and $\tilde{C}_{\phi \phi}\left(k_{0}\right)>0$ is equivalent to $T^{*}>T^{*}\left(\zeta ; k_{0}\right)$ (see (19) and below). The most probable eigenmode corresponds to $k_{b}$ such that $\tilde{C}_{\alpha \beta}^{0}(k)$ assumes a minimum at $k=k_{b}$. Hence, the instantaneous uniform state occurs with a higher probability than the periodic instantaneous state with infinitesimal amplitude and any wavelength for $\tilde{C}_{\phi \phi}\left(k_{b}\right)>0$, i.e. for $T^{*}>T^{*}\left(\zeta ; k_{b}\right)$. When for given $\zeta$ temperature decreases from $T^{*}=T^{*}\left(\zeta ; k_{b}\right)$, the range of $k$ such that $\bar{p}\left[\Phi_{i} \tilde{g}_{i}(\mathbf{k} \mid k), 0\right] / p[0,0]>1$ increases. This means that the population of the instantaneous periodic states that occur more frequently than the instantaneous uniform state increases. Ordering of the significant fraction of the instantaneous states can be recognized as a pretransitional effect associated with the transition to the corresponding ordered 
phase. The line in the phase diagram corresponding to $\tilde{C}_{\phi \phi}^{0}\left(k_{b}\right)=0$ can be identified with the structural line, because different instantaneous states dominate on different sides of this line. Note that the structural line coincides precisely with the boundary of stability of the uniform phase in the MF approximation (MF spinodal).

Near the minimum of $\tilde{C}_{\alpha \beta}^{0}(k)$ the probability ratio in Eq.(41) varies slowly with $k$. Further studies are required to determine the probability that different wave packages corresponding to $k \approx k_{b}$ are thermally excited, and to verify if the wave packages are related to clusters of ions in real space.

We should note that the large-amplitude periodic states may be more probable than the uniform state even for $\tilde{C}_{\alpha \beta}^{0}\left(k_{b}\right)>0$, if the term $O\left(\Phi_{j}\right)$ in Eq.(41) is negative. Note also that in the above studies we assumed that the instantaneous states did not include the eigenmode $\eta$ associated with the larger eigenvalue of $\tilde{C}_{\alpha \beta}^{0}(k)$. Interesting information on pretransitional effects can be gained from studies of the effect of coupling between the two eigenmodes in $\Omega_{\text {int }}$. In particular, the probabilites of the eigenmodes $\eta$, and $\phi$, given by $\int D \phi \bar{p}[\phi, \eta]$ and $\int D \eta \bar{p}[\phi, \eta]$ respectively, are of considerable interest. In the case of the RPM [57] the charge-charge correlations play a role analogous to short-range interactions between the ions [1, 57, 58], and at low volume fractions $\int D \phi \bar{p}[\phi, \eta]$ turnes out to be important for the phase separation [41, 57]. The above questions go beyond the scope of this work.

\section{EIGENMODES AND MF BOUNDARY OF STABILITY OF THE DISOR- DERED PHASE IN THE GENERAL CASE OF ARBITRARY $\delta$ AND $\nu$}

\section{A. Eigenmodes}

Our purpose here is to find the instantaneous states that may lead to instabilities of the disordered phase for arbitrary $\delta$ and $\nu$ in the MF. In order to find the spinodal lines in the MF (structural lines), it is sufficient to analyze $\beta \Omega_{2}$ (see Eqs.(18) and (39)). $\tilde{C}_{\alpha \beta}^{0}(k)$ (Eq.(19) ) can be diagonalized, and $\Omega_{2}$ can be written in the form

$$
\beta \Omega_{2}=\frac{1}{2} \int \frac{d \mathbf{k}}{(2 \pi)^{2}}\left[\tilde{\phi}(\mathbf{k}) \tilde{C}_{\phi \phi}^{0}(k) \tilde{\phi}(-\mathbf{k})+\tilde{\eta}(\mathbf{k}) \tilde{C}_{\eta \eta}^{0}(k) \tilde{\eta}(-\mathbf{k})\right] .
$$

Both $\tilde{C}_{\phi \phi}^{0}(k)$ and $\tilde{C}_{\eta \eta}^{0}(k)$ depend on the wavenumber $k$ in a nontrivial way,

$$
\tilde{C}_{\phi \phi}^{0}(k)=\frac{\tilde{C}_{++}^{0}(k)+\tilde{C}_{--}^{0}(k)-\operatorname{sign}\left(\tilde{C}_{+-}^{0}(k)\right) \mathcal{B}(k)}{2}
$$


and

$$
\tilde{C}_{\eta \eta}^{0}(k)=\frac{\tilde{C}_{++}^{0}(k)+\tilde{C}_{--}^{0}(k)+\operatorname{sign}\left(\tilde{C}_{+-}^{0}(k)\right) \mathcal{B}(k)}{2},
$$

where

$$
\mathcal{B}(k)=\sqrt{\mathcal{A}^{2}+4 \tilde{C}_{+-}^{0}(k)^{2}},
$$

and

$$
\mathcal{A}(k)=\operatorname{sign}\left(\tilde{C}_{+-}^{0}(k)\right)\left[\tilde{C}_{--}^{0}(k)-\tilde{C}_{++}^{0}(k)\right] .
$$

In the above $\tilde{C}_{\alpha \beta}^{0}$ are given in Eq.(19) $), a_{\alpha \beta}$ are given in Appendix A and $\tilde{V}_{\alpha \beta}(k)$ are given in Eqs.(21)-(23).

In the general case the corresponding eigenmodes have the forms

$$
\begin{aligned}
& \tilde{\phi}(\mathbf{k})=\tilde{a}(k) \Delta \tilde{\rho}_{+}^{*}(\mathbf{k})-\tilde{b}(k) \Delta \tilde{\rho}_{-}^{*}(\mathbf{k}), \\
& \tilde{\eta}(\mathbf{k})=\tilde{b}(k) \Delta \tilde{\rho}_{+}^{*}(\mathbf{k})+\tilde{a}(k) \Delta \tilde{\rho}_{-}^{*}(\mathbf{k}),
\end{aligned}
$$

with

$$
\begin{gathered}
\tilde{a}(k)=\left[\frac{\mathcal{A}(k)+\mathcal{B}(k)}{2 \mathcal{B}(k)}\right]^{1 / 2}, \\
\tilde{b}(k)=\left[\frac{-\mathcal{A}(k)+\mathcal{B}(k)}{2 \mathcal{B}(k)}\right]^{1 / 2} .
\end{gathered}
$$

The eigenmodes represent two order-parameter (OP) fields, and in principle either one of them may lead to instability of the disordered phase.

On the MF level the FT analogs (31) of the OZ equations are decoupled in the eigenbasis of the vertex functions, i.e.

$$
\tilde{G}_{\phi \phi}^{0}(k)=1 / \tilde{C}_{\phi \phi}^{0}(k) \quad \text { and } \quad \tilde{G}_{\eta \eta}^{0}(k)=1 / \tilde{C}_{\eta \eta}^{0}(k) .
$$

Note that in the fully symmetrical case $(\nu=\delta=0), \tilde{C}_{--}^{0}(k)=\tilde{C}_{++}^{0}(k)$, and $\tilde{a}(k)=\tilde{b}(k)=$ $1 / \sqrt{2}$, hence $\phi$ and $\eta$ are proportional to the charge- and the number density respectively. Moreover, Eqs.(43) and (44) reduce to $\tilde{C}_{\phi \phi}^{0}(k)=\left(\tilde{C}_{++}^{0}(k)+\tilde{C}_{--}^{0}(k)-2 \tilde{C}_{+-}^{0}(k)\right) / 2$ and $\tilde{C}_{\eta \eta}^{0}(k)=\left(\tilde{C}_{++}^{0}(k)+\tilde{C}_{--}^{0}(k)+2 \tilde{C}_{+-}^{0}(k)\right) / 2$, representing the charge-charge and the densitydensity vertex function respectively. When any asymmetry is present and $\tilde{C}_{--}^{0}(k) \neq \tilde{C}_{++}^{0}(k)$, $C_{\phi \phi}^{0}$ and $C_{\eta \eta}^{0}$ that satisfy the decoupled OZ equations differ from the charge- and number density vertex functions respectively, in agreement with Ref.[1]. 


\section{B. Dominant order parameters}

Let us focus on thermodynamic conditions such that the uniform distributions $\rho_{0 \alpha}^{*}$ correspond to the minimum of $\Omega^{M F}$, and consider the two OP fields with equal wavelengths and equal infinitesimal amplitudes. The field leading to a smaller increase of $\Omega^{M F}$ is thermally excited with a higher probability (7), and such OP dominates over the other one. Which one of the two infinitesimal fields, $\tilde{\phi}(\mathbf{k})$ or $\tilde{\eta}(\mathbf{k})$ dominates, depends on which function, $\tilde{C}_{\phi \phi}(k)$ or $\tilde{C}_{\eta \eta}(k)$, is smaller (see (42) $)$. This, in turn, depends on $\operatorname{sign}\left(\tilde{C}_{+-}(k)\right)$ (see (43) and (44) $)$. From Eqs.(19) and (23) we obtain the line

$$
T^{*}=\frac{4 \pi \cos k}{k^{2}} a_{+-}^{-1}
$$

separating the phase space $\left(\zeta, T^{*}\right)$ into the high-temperature part where the OP $\tilde{\phi}(\mathbf{k})$ dominates, and the low-temperature part where the other eigenmode dominates. In the above $a_{+-}$is a function of $\zeta$ (or $s$ ) given in Appendix A. Note that for different wavenumbers $k$ Eq.(52) yields quite different lines. In particular, for long-wavelengths $k \rightarrow 0$, corresponding to phase separation into two uniform phases, from (52) we find that $\tilde{\eta}(\mathbf{0})$ dominates for all temperatures $T^{*}<\infty$. We can thus conclude that if the phase separation (stable or metastable) occurs, it is induced by the field $\eta$. On the other hand, for $k \geq \pi / 2$, corresponding to the periodic ordering of ions with the wavelength $2 \pi / k \leq 4$ (in $\sigma_{+-}$units), the OP $\tilde{\phi}(\mathbf{k})$ dominates for all temperatures $T^{*}>0$. In principle, the uniform system may become unstable with respect to the eigenmodes with $0<k<\pi / 2$ as well. As we show later, this is indeed the case for very large asymmetry. In such a system $\tilde{\phi}(\mathbf{k})$ or $\tilde{\eta}(\mathbf{k})$ dominates for temperatures higher or lower than the temperature given in Eq.(52) respectively.

\section{The MF spinodal lines}

Let us first analyze the stability of the uniform phase with respect to long-wavelength deviations from homogeneous distributions of ions in the MF approximation. For $k \rightarrow 0$ Eqs.(44) and (43) assume the asymptotic forms

$$
\tilde{C}_{\eta \eta}^{0}(k)=\frac{a_{++} Z^{-1}+a_{--} Z+2 a_{+-}-4 \pi \beta^{*} \delta^{2}}{Z+Z^{-1}}+O\left(k^{2}\right),
$$

and

$$
\tilde{C}_{\phi \phi}^{0}(k)=\frac{4 \pi \beta^{*}\left(Z+Z^{-1}\right)}{k^{2}}+O(1)
$$


$\tilde{C}_{\phi \phi}^{0}(k)$ diverges for $k \rightarrow 0$. When $\tilde{C}_{\phi \phi}^{0}(0)=\infty$, then the probability $p \propto \exp \left(-\beta \Omega^{M F}[\phi, \eta]\right)$ of thermally exciting any nonzero $\tilde{\phi}(0)$ vanishes (see (17) and (42)), as required by the global charge neutrality. $\tilde{C}_{\eta \eta}^{0}(k)$ can vanish for $k=0$ when

$$
T^{*}=\frac{4 \pi \delta^{2}}{a_{++} Z^{-1}+a_{--} Z+2 a_{+-}} .
$$

The above equation describes the MF spinodal line associated with the phase separation into two uniform phases characterized by different values of the OP $\eta$.

The MF instability with respect to the $k$-mode is given by

$$
\operatorname{det} \tilde{C}_{\alpha \beta}^{0}(k)=\tilde{C}_{\eta \eta}^{0}(k) \tilde{C}_{\phi \phi}^{0}(k)=0,
$$

where

$$
\begin{array}{r}
\tilde{C}_{\eta \eta}^{0}(k) \tilde{C}_{\phi \phi}^{0}(k)=\left(a_{++}+\beta \tilde{V}_{++}(k)\right)\left(a_{--}+\beta \tilde{V}_{--}(k)\right)-\left(a_{+-}+\beta \tilde{V}_{+-}(k)\right)^{2}= \\
-\frac{\sin ^{2}(k \delta)}{k^{4}}\left(4 \pi \beta^{*}\right)^{2}+\frac{b(k, s, \delta, \nu)}{k^{2}} 4 \pi \beta^{*}+d(s, \delta, \nu),
\end{array}
$$

and the functions $b(k, s, \delta, \nu)$ and $d(s, \delta, \nu)>0$ are given in Appendix B.

Note that there is a single positive solution of Eq.(56) for $\beta^{*}$ for all values of the remaining parameters. The wavector at the MF spinodal (structural line) is determined from

$$
\partial\left(\tilde{C}_{\eta \eta}^{0}(k) \tilde{C}_{\phi \phi}^{0}(k)\right) / \partial k=0 .
$$

The solutions of Eqs. (56) and (58) give the spinodal line and the wavenumber of the dominant instantaneous state.

\section{Nature of the eigenmodes for different $\delta$ and $\nu$}

It is instructive to discuss the nature of the eigenmodes that may drive the system out of the disordered state. The equations (47) and (48) can be easily inverted to give

$$
\begin{aligned}
\Delta \tilde{\rho}_{+}^{*}(\mathbf{k}) & =\tilde{a}(k) \tilde{\phi}(\mathbf{k})+\tilde{b}(k) \tilde{\eta}(\mathbf{k}) \\
\Delta \tilde{\rho}_{-}^{*}(\mathbf{k}) & =-\tilde{b}(k) \tilde{\phi}(\mathbf{k})+\tilde{a}(k) \tilde{\eta}(\mathbf{k}) .
\end{aligned}
$$

The local charge density,

$$
q(\mathbf{r})=Z \Delta \rho_{+}^{*}(\mathbf{r})-\Delta \rho_{-}^{*}(\mathbf{r})
$$


(in $\left|e_{-}\right| \sigma_{+-}^{-3}$ units), and the local deviation of the number density of ions from the most probable value (in $\sigma_{+-}^{-3}$ units),

$$
\Delta \rho(\mathbf{r})=\Delta \rho_{+}^{*}(\mathbf{r})+\Delta \rho_{-}^{*}(\mathbf{r})
$$

are related to the two OP's by

$$
\begin{gathered}
\tilde{q}(\mathbf{k})=(Z \tilde{a}(k)+\tilde{b}(k)) \tilde{\phi}(\mathbf{k})+(Z \tilde{b}(k)-\tilde{a}(k)) \tilde{\eta}(\mathbf{k}) \\
\Delta \tilde{\rho}(\mathbf{k})=(\tilde{a}(k)-\tilde{b}(k)) \tilde{\phi}(\mathbf{k})+(\tilde{a}(k)+\tilde{b}(k)) \tilde{\eta}(\mathbf{k}) .
\end{gathered}
$$

The inverse relations are

$$
\begin{aligned}
& \tilde{\phi}(\mathbf{k})=\frac{1}{Z+1}[(\tilde{a}(k)+\tilde{b}(k)) \tilde{q}(\mathbf{k})+(\tilde{a}(k)-Z \tilde{b}(k)) \Delta \tilde{\rho}(\mathbf{k})] \\
& \tilde{\eta}(\mathbf{k})=\frac{1}{Z+1}[(\tilde{b}(k)-\tilde{a}(k)) \tilde{q}(\mathbf{k})+(Z \tilde{a}(k)+\tilde{b}(k)) \Delta \tilde{\rho}(\mathbf{k})] .
\end{aligned}
$$

On the low-temperature side of the structural line the dominant instantaneous states consist of the charge- and the number-density waves with the wavnumber $k_{b}$ and the amplitudes that satisfy the relation

$$
\Delta \tilde{\rho}\left(\mathbf{k}_{b}\right)=R \tilde{q}\left(\mathbf{k}_{b}\right)
$$

As seen from Eqs.(63) - (65) $0)$, corresponds to

$$
R=\frac{\left(\tilde{a}\left(k_{b}\right)-\tilde{b}\left(k_{b}\right)\right)}{\left(Z \tilde{a}\left(k_{b}\right)+\tilde{b}\left(k_{b}\right)\right)} .
$$

For $\tilde{a}\left(k_{b}\right)=\tilde{b}\left(k_{b}\right)$, i.e. in the RPM limit, $R=0$, and $\tilde{\phi}\left(k_{b}\right) \propto \tilde{q}\left(\mathbf{k}_{b}\right)$. When the instability of $\Omega^{M F}$ is induced by $\tilde{\eta}\left(\mathbf{k}_{b}\right)$ and the other OP vanishes, then

$$
R=\frac{\left(\tilde{a}\left(k_{b}\right)+\tilde{b}\left(k_{b}\right)\right)}{\left(Z \tilde{b}\left(k_{b}\right)-\tilde{a}\left(k_{b}\right)\right)} .
$$

For $Z \tilde{b}\left(k_{b}\right)=\tilde{a}\left(k_{b}\right)$ we have $1 / R=0$, and $\tilde{\eta}\left(\mathbf{k}_{b}\right) \propto \Delta \tilde{\rho}\left(\mathbf{k}_{b}\right)$.

For $R \ll 1$ the charge-density waves dominate over the number-density waves in the dominant eigenmode. In such a case the regions of the excess positive charge are followed by regions of excess negative charge. The number densities in the charged regions are comparable. For $R \gg 1$ the number-density waves dominate, i.e. regions with excess number-density are followed by regions containing a smaller number of ions. Both the dense and the dilute regions are nearly charge-neutral for $R \gg 1$. 
In real-space representation the OP fields are given by convolutions,

$$
\phi(\mathbf{r})=\int d \mathbf{r}_{1}\left[\Delta \rho_{+}^{*}\left(\mathbf{r}_{1}\right) a\left(\mathbf{r}-\mathbf{r}_{1}\right)-\Delta \rho_{-}^{*}\left(\mathbf{r}_{1}\right) b\left(\mathbf{r}-\mathbf{r}_{1}\right)\right],
$$

with analogous expression for the field $\eta(\mathbf{r})$, where $a(\mathbf{r})$ and $b(\mathbf{r})$ are inverse Fourier transforms of the functions defined in Eq.(49) and (50).

\section{CASE OF EQUAL SIZES}

\section{A. MF spinodal and the wavevector of the most probable instantaneous state}

The phase separation (55) occurs only for different sizes of the ionic species $(\delta \neq 0)$. In the size-symmetric case the disordered phase is unstable only with respect to periodic ordering in MF. Let us consider the periodic ordering, for which the MF spinodal is given by Eqs. (56) and (58). For $\delta=0$ we obtain

$$
a_{\alpha \alpha}=\frac{1}{\rho_{0 \alpha}^{*}}+a_{+-}
$$

and

$$
a_{+-}=\frac{\pi(4-s)\left(2+s^{2}\right)}{(1-s)^{4}} .
$$

The boundary of stability of the disordered phase and the associated wavevector reduce to the form

$$
T_{R}^{*}(s)=-\frac{24 \cos k_{R}}{k_{R}^{2}} s, \quad \tan k_{R}=-\frac{2}{k_{R}} .
$$

The above form is identical to the one found for the RPM within the same approach[3, 52]. The subscript $R$ indicates that the MF spinodal and the wavenumber refer to the RPM. The charge asymmetry has no effect on the boundary of stability of the disordered phase on the MF level of our theory, as long as the sizes are the same. This result agrees with MF predictions of other theories [17, 59, 60, 61]. Beyond MF this property does not persist [17, 60, 61] due to the coupling between the two OP's in $\Omega_{\text {int }}$.

\section{B. Eigenmodes}

In this case we obtain the following form of Eq.(46)

$$
\mathcal{A}=-\operatorname{sign} \tilde{C}_{+-}(k) \frac{4 \nu}{1-\nu^{2}}\left(\frac{\pi}{6 s}+\frac{4 \pi \beta^{*} \cos k}{k^{2}}\right) .
$$


At the MF spinodal line (720) $\mathcal{A}=0$, and in turn $\tilde{a}\left(k_{R}\right)=\tilde{b}\left(k_{R}\right)$ (see Eqs.(49) and (150)), thus

$$
\begin{aligned}
& \tilde{\phi}\left(\mathbf{k}_{R}\right)=\frac{1}{\sqrt{2}}\left(\Delta \tilde{\rho}_{+}\left(\mathbf{k}_{R}\right)-\Delta \tilde{\rho}_{-}\left(\mathbf{k}_{R}\right)\right) \\
& \tilde{\eta}\left(\mathbf{k}_{R}\right)=\frac{1}{\sqrt{2}}\left(\Delta \tilde{\rho}_{+}\left(\mathbf{k}_{R}\right)+\Delta \tilde{\rho}_{-}\left(\mathbf{k}_{R}\right)\right) .
\end{aligned}
$$

These relations are the same as in the RPM, although for $Z \neq 1 \tilde{\phi}(\mathbf{k})$ differs from the charge-density amplitude (61). Beyond the spinodal line $\mathcal{A} \neq 0$, and the eigenmodes contain different proportions of $\Delta \tilde{\rho}_{+}$and $\Delta \tilde{\rho}_{-}$.

\section{THE MF SPINODAL ASSOCIATED WITH THE GAS-LIQUID PHASE SEP- ARATION}

\section{A. Eigenmodes in the case of $k \rightarrow 0$}

Let us discuss the nature of the eigenmodes for $k \rightarrow 0$. From Eqs. (49) and (50) we find for arbitrary $\delta$ and $\nu$

$$
\tilde{a}(0)=\frac{Z}{\sqrt{1+Z^{2}}}, \quad \tilde{b}(0)=\frac{1}{\sqrt{1+Z^{2}}} .
$$

In the long-wavelength limit the amplitudes of the eigenmodes assume the forms

$$
\begin{gathered}
\tilde{\eta}(0)=\frac{1}{\sqrt{1+Z^{2}}}\left(\Delta \tilde{\rho}_{+}^{*}(0)+Z \Delta \tilde{\rho}_{-}^{*}(0)\right) \\
\tilde{\phi}(0)=\frac{1}{\sqrt{1+Z^{2}}}\left(Z \Delta \tilde{\rho}_{+}^{*}(0)-\Delta \tilde{\rho}_{-}^{*}(0)\right) .
\end{gathered}
$$

Note that from Eqs.(178) and (61) it follows that $\tilde{\phi}(0) \propto \tilde{q}(0)$. The charge-neutrality condition (6) is equivalent to $\tilde{\phi}(0)=0$. For $\tilde{\phi}(0)=0$ we obtain

$$
\tilde{\eta}(0)=\frac{\sqrt{1+Z^{2}}}{1+Z} \Delta \tilde{\rho}(0) .
$$

In sec.IIIB we have shown that for $k \rightarrow 0$ the dominant eigenmode is $\tilde{\eta}(0)$. Eq.(79) shows that $\tilde{\eta}(0) \propto \Delta \tilde{\rho}(0)$. Hence, we find the usual phase separation into ion-dilute and ion-dense phases for all values of the asymmetry parameters, as expected. 


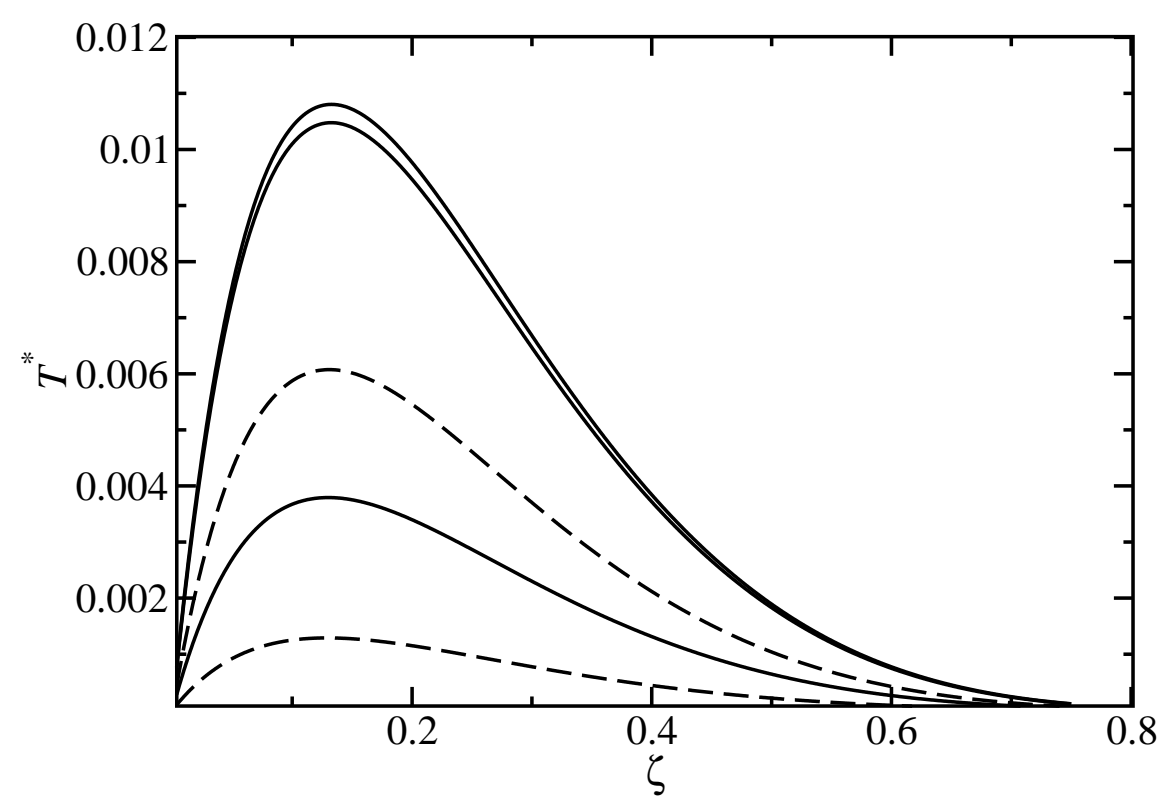

FIG. 1: MF spinodal lines for the gas-liquid separation for $\delta=0.2(\lambda=3 / 2)$. The solid and the dashed lines correspond to $\nu \geq 0$ and $\nu<0$ respectively. From the top to the bottom, the solid lines correspond to $\nu=0.5, \nu=0$ and $\nu=0.9$. The upper and the lower dashed line correspond to $\nu=-0.5$ and $\nu=-0.9$ respectively.

\section{B. MF Spinodal lines}

In our MF approximation the spinodal line for the gas-liquid separation assumes a single maximum $T_{c}^{*}\left(\zeta_{c}\right)$ at the critical point (cp) for any pair of $\delta \neq 0$ and $\nu$. The MF spinodal lines are shown in Figs.1,2 and 3 for $\delta=0.2, \delta=0,6$ and $\delta=0.9$ respectively, for several values of $\nu$. The spinodal lines assume the characteristic asymmetric shape as in the RPM [1, 2]. The critical temperature and the volume fraction, $T_{c}^{*}$ and $\zeta_{c}$ respectively, are shown in Fig.4 as functions of the size asymmetry for several charge ratios. For a given charge ratio the dependence of $\zeta_{c}$ on the size asymmetry agrees qualitatively with the major trends found in Monte Carlo (MC) simulations [18, 19, 20, 22, 44]. Namely, $\zeta_{c}$ is a convex function of $\delta$, and its value is of the same order of magnitude as in Ref. [22]. In contrast, $T_{c}^{*}$ is a convex function of $\delta$ for fixed $\nu$, whereas in MC a concave function was obtained [18, 19, 20, 22]. For large asymmetries the value of $T_{c}^{*}$ is overestimated, whereas for $\delta=0$ we have $T_{c}^{*}=0$ in our MF approximation. On the other hand, the dependence of $T_{c}^{*}$ on $\nu$ for fixed $\delta$ agrees qualitatively with MC results [22]. Namely, for $\delta<0$ the $T_{c}^{*}$ decreases with increasing charge asymmetry, and for $\delta>0$ the lines $T_{c}^{*}(\delta)$ corresponding to different charge ratios intersect 


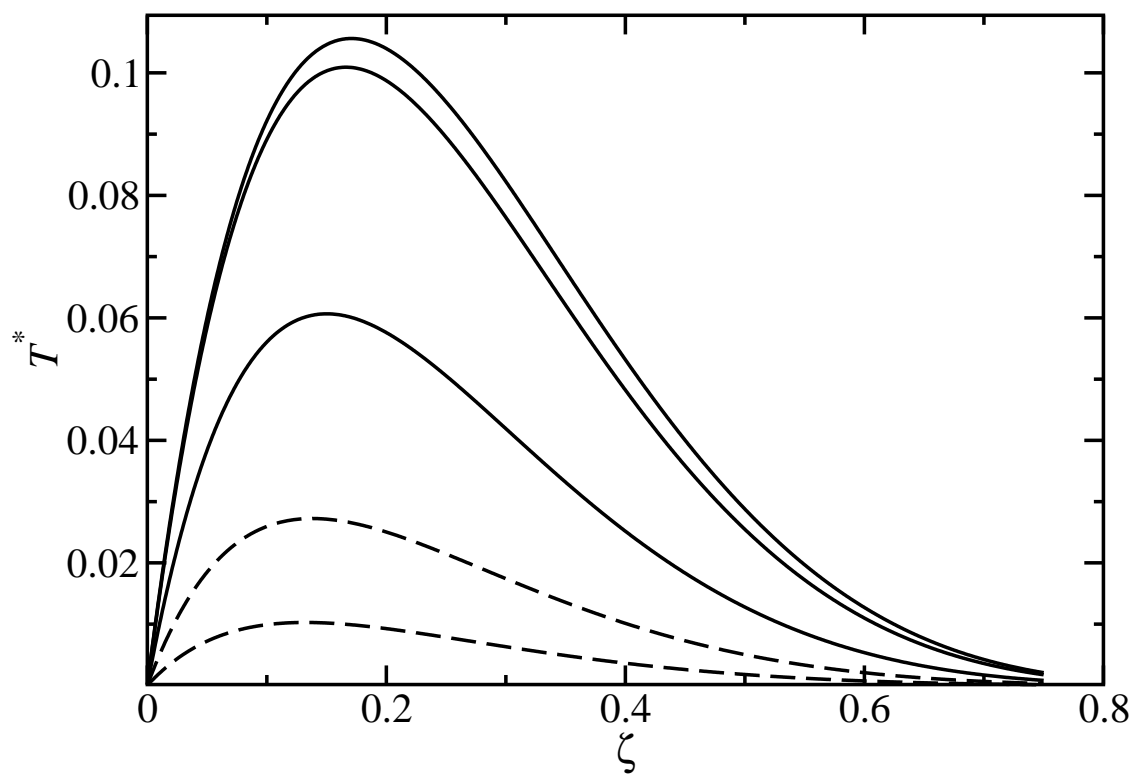

FIG. 2: MF spinodal lines for the gas-liquid separation for $\delta=0.6(\lambda=4)$. The solid and the dashed lines correspond to $\nu \geq 0$ and $\nu<0$ respectlively. From the top to the bottom, the solid lines correspond to $\nu=0.9, \nu=0.5$ and $\nu=0$. The upper and the lower dashed line correspond to $\nu=-0.5$ and $\nu=-0.8$ respectively.

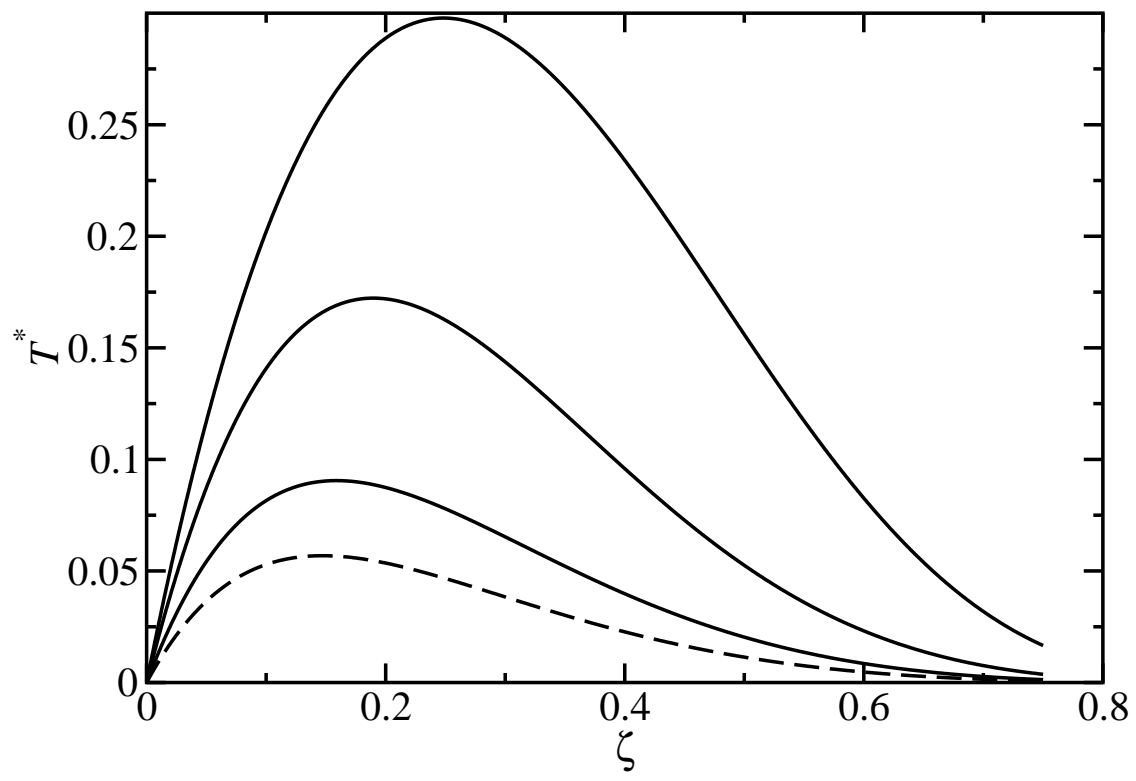

FIG. 3: MF spinodal lines for the gas-liquid separation for $\delta=0.9(\lambda=19)$. The solid and the dashed lines correspond to $\nu \geq 0$ and $\nu<0$ respectlively. From the top to the bottom, the solid lines correspond to $\nu=0.9, \nu=0.5$ and $\nu=0.0$. The dashed line corresponds to $\nu=-0.3$. 
each other (Fig.4 (upper panel) here and Fig.9 in Ref. [22]). In the case of $\zeta_{c}$, a correct dependence on the charge asymmetry is found for $\delta>0$, whereas for $\delta<0$ our results do not agree with (partial) results obtained in Ref. [22]).

In the case of $\delta=1$ and $\nu \rightarrow 1(\lambda=\infty, Z \rightarrow \infty)$, and for $\zeta=O\left(Z^{0}\right)$, Eq. (55) assumes the simple form

$$
T^{*}=3 \zeta(1-\zeta)^{2}
$$

The critical-point temperature, $T_{c}^{*}=4 / 9$, and density, $\zeta_{c}=1 / 3$, are both large compared to the corresponding values in the RPM, in contrast to the simulation results. Thus, some of the trends found in simulations are correctly predicted by the MF theory, while some other trends are not. Note, however that beyond MF coupling between the two OP's may have a significant effect on the spinodal. In particular, beyond $\mathrm{MF}_{c}^{*}>0$ is found for $\delta=0[\underline{3}, \underline{52}]$ as a result of the coupling between the two OP's in $\Omega_{\text {int }}$ (see Eq.(25) ). Significant effect of the coupling between the two OP's on the spinodal should be expected also for $\delta \neq 0$ and $\nu \neq 0$. Indeed, a qualitatively correct dependence of the location of the cp on $\nu$ is found for $\delta=0$ in the CV approach [17], beyond MF. As mentioned in sec.IIA, the CV approach and our mesoscopic theory are closely related [49]. Correct trends are also found when ion pairing is explicitly taken into account [15, 16].

\section{THE MF SPINODAL ASSOCIATED WITH PERIODIC ORDERING OF IONS}

The structural line (MF spinodal) associated with the periodic ordering of the instantaneous states, as well as the most probable periodic structures depend qualitatively on $\delta$ and $\nu$. The key role is played by the size asymmetry. Three regimes of the size asymmetry can be distinguished, although there are no sharp boundaries, but rather smooth crossovers between them. The approximate ranges of the first two regimes are $\delta<0.4(\lambda<2.3)$, and $0.4<\delta<0.9(2.3<\lambda<19)$. The qualitative dependence on $\nu$ is found in the second regime only. Third regime corresponds to the asymptotic case of $\delta, \nu \rightarrow 1$, i.e. to extreme chargeand size asymmetry. In the other (unphysical) extreme, $\delta, \nu \rightarrow-1$, the periodic ordering is suppressed. Detailed description of each case is given in the following subsections. 

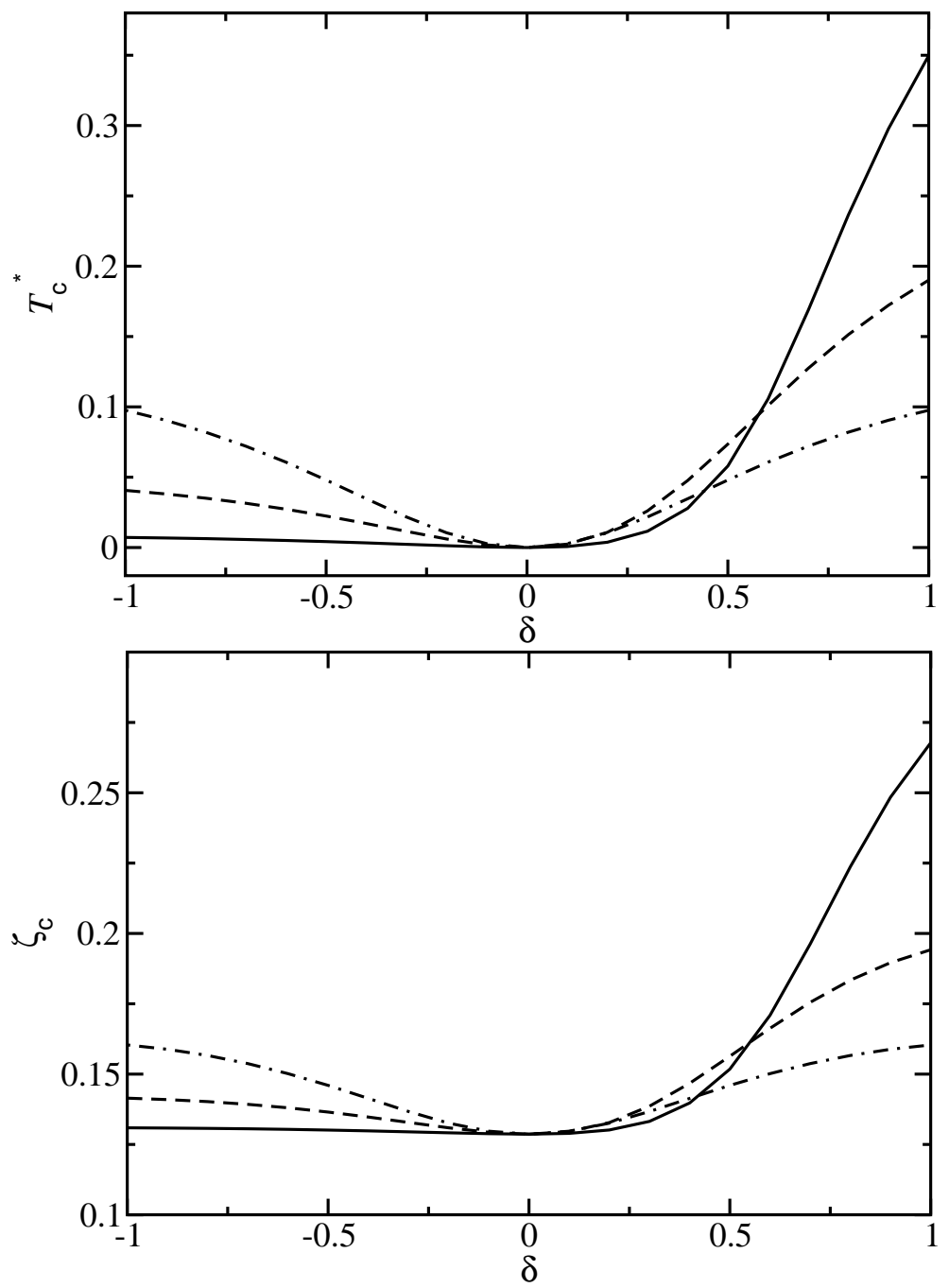

FIG. 4: Critical temperature (top) and critical volume fraction (bottom) as functions of the size $\operatorname{asymmetry} \delta$, for three values of the charge asymmetry $\nu$. Solid, dashes and dash-dotted lines correspond to $\nu=0.9,0.5$ and 0 respectively.

\section{A. Small size asymmetry}

1. MF spinodal lines and wavevectors in the dominant instantaneous states

The MF spinodal in the case of the small size-asymmetry $\delta<0.4(\lambda<2.3)$ is qualitatively the same as in the RPM, i.e. along the spinodal $T^{*}$ is a monotonically increasing function of $\zeta$ (Fig.5). Somewhat surprising is the fact that for larger charge-asymmetry the ordering occurs at higher temperatures. It is not clear whether this tendency persists beyond MF. The wavenumber characterizing the period of the oscillations of the OP field in the ordered 


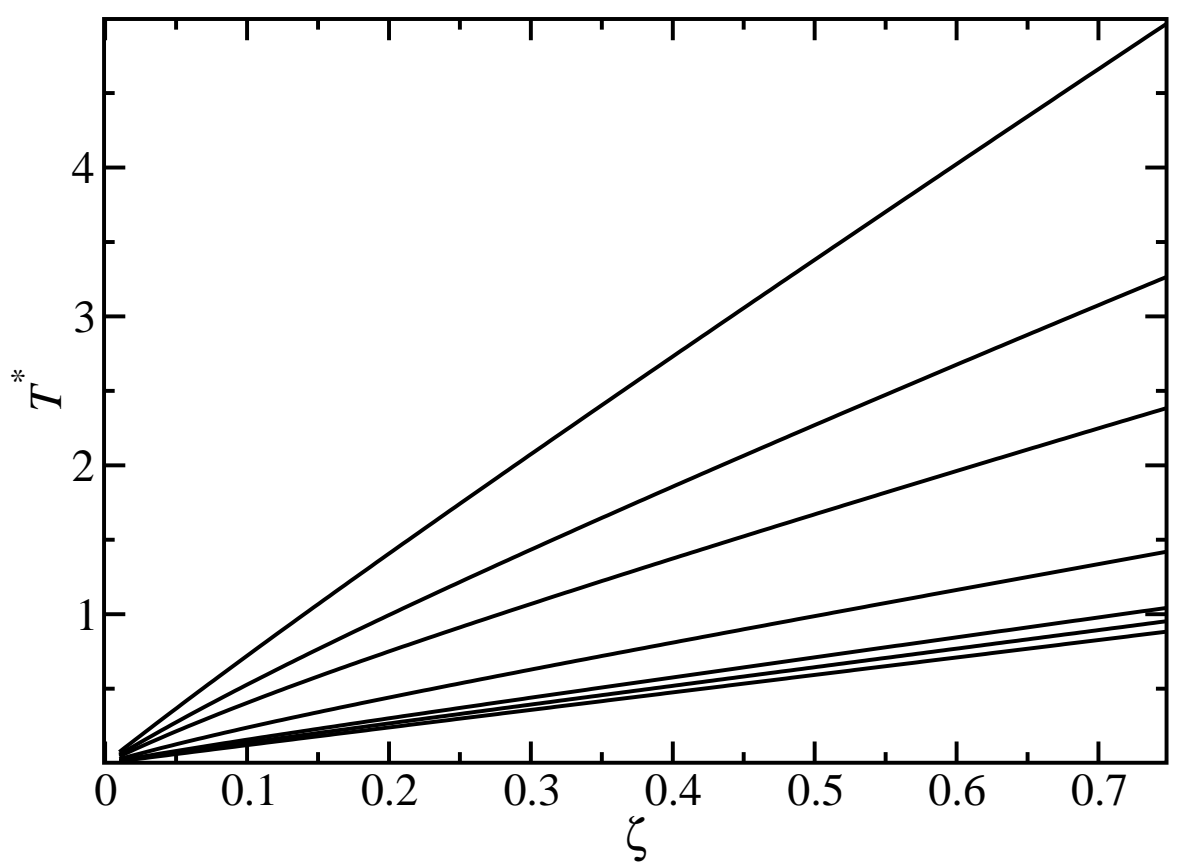

FIG. 5: MF spinodal lines for the transition to the ordered phase for $\delta=0.2$. From the top to the bottom lines $\nu=0.9, \nu=0.7, \nu=0.5 \nu=0.0, \nu=-0.5, \nu=-0.7$ and $\nu=-0.9$. Temperature $T^{*}$ and the volume fraction of ions $\zeta$ are in dimensionless reduced units defined in Eqs.(11) and (12) respectively.

phase is $k_{b}>\pi / 2$, and its value is almost independent of $\zeta$ (see Fig.6). The size of the regions of increased or depleted densities of the two ionic species, $\sim \pi / k_{b}<\left(\sigma_{+}+\sigma_{-}\right)$, is comparable to the size of the ions, and the ordered phase should be identified with a standard 'hard' ionic crystal. According to the discussion in sec.III.B, the relevant OP field is $\tilde{\phi}\left(k_{b}\right)$ for the whole temperature range. The amplitudes of the number- and the charge-density waves in the dominant instantaneous state are related according to Eqs.(66)-(67). The amplitude ratios $R$ for $\delta=0.2$ are shown in Fig.7.

The above results indicate that the behavior of the PM for $\delta<0.4$ is qualitatively the same as in the RPM. By analogy with the RPM, we expect that the transition to the ionic crystal is fluctuation-induced first-order, and occurs at significantly lower $T^{*}$ and higher $\zeta$ compared to the structural line [37]. 


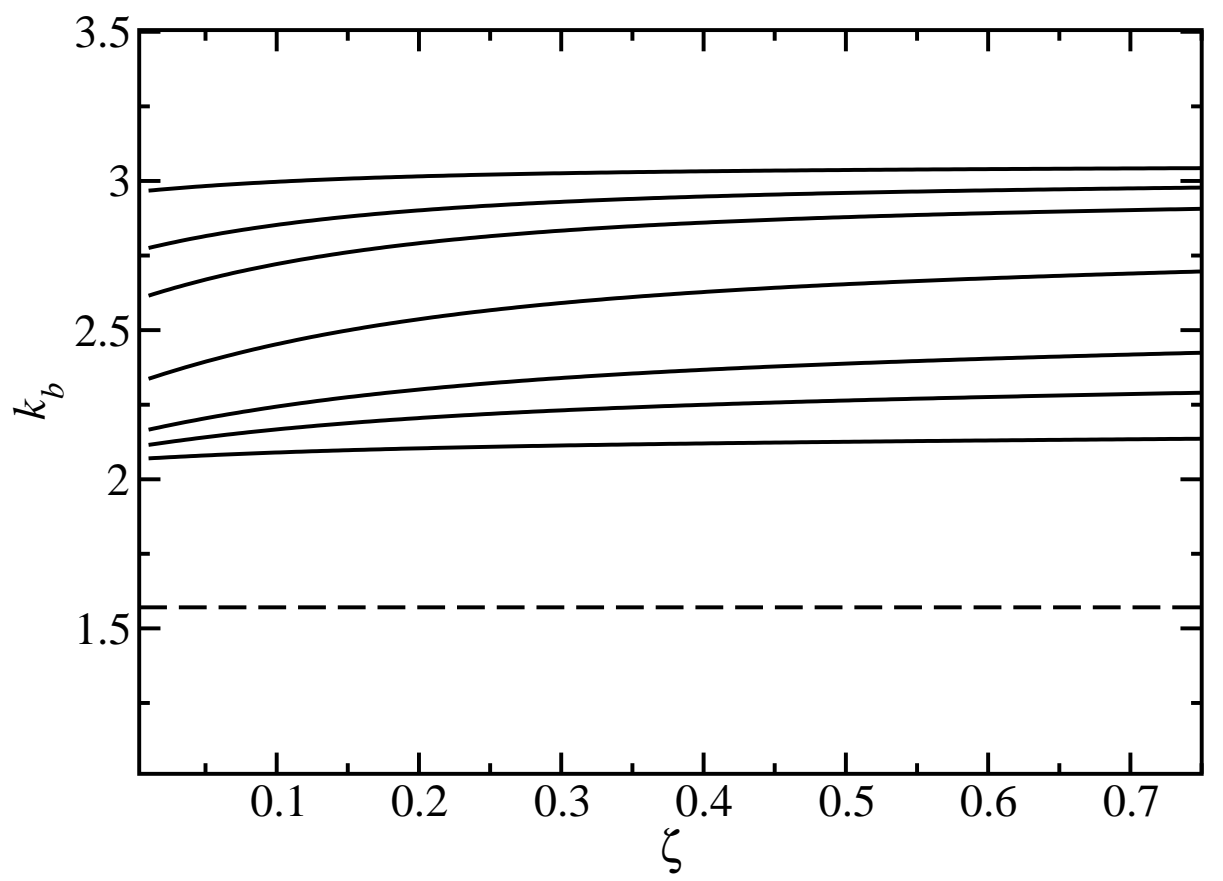

FIG. 6: The wavenumber $k_{b}$ corresponding to the ordering of ions along the MF spinodal lines shown in Fig.5 for $\delta=0.2$. From the bottom to the top lines $\nu=0.9, \nu=0.7, \nu=0.5 \nu=0.0$, $\nu=-0.5, \nu=-0.7$ and $\nu=-0.9 . k_{b}$ is in $\sigma_{+-}^{-1}$ units and $\zeta$ is the volume fraction of ions.

\section{Eigenmodes for very small asymmetries}

Let us determine the behavior of the eigenmodes in the RPM limit. For $\delta, \nu \rightarrow 0$ we find from Eq.(46) that $\mathcal{A} \rightarrow 0$, and

$$
\begin{aligned}
& \tilde{a}(k) \simeq_{\delta, \nu \rightarrow 0} \frac{1}{\sqrt{2}}(1+y)+O\left(\delta^{2}, \delta \nu, \nu^{2}\right) \\
& \tilde{b}(k) \simeq_{\delta, \nu \rightarrow 0} \frac{1}{\sqrt{2}}(1-y)+O\left(\delta^{2}, \delta \nu, \nu^{2}\right)
\end{aligned}
$$

where

$$
y=\frac{\mathcal{A}}{2 \mathcal{B}}=O(\delta)+O(\nu)
$$

The eigenmodes (47) and (48) take the forms

$$
\begin{array}{r}
\tilde{\phi}(\mathbf{k})=\frac{1}{\sqrt{2}}\left[\Delta \tilde{\rho}_{+}(\mathbf{k})-\Delta \tilde{\rho}_{-}(\mathbf{k})+y\left(\Delta \tilde{\rho}_{+}(\mathbf{k})+\Delta \tilde{\rho}_{-}(\mathbf{k})\right)\right]+O\left(\delta^{2}, \delta \nu, \nu^{2}\right) \\
=\frac{1}{\sqrt{2}}[(1-\nu) \tilde{q}(\mathbf{k})+(y-\nu) \Delta \tilde{\rho}(\mathbf{k})]+O\left(\delta^{2}, \delta \nu, \nu^{2}\right)
\end{array}
$$




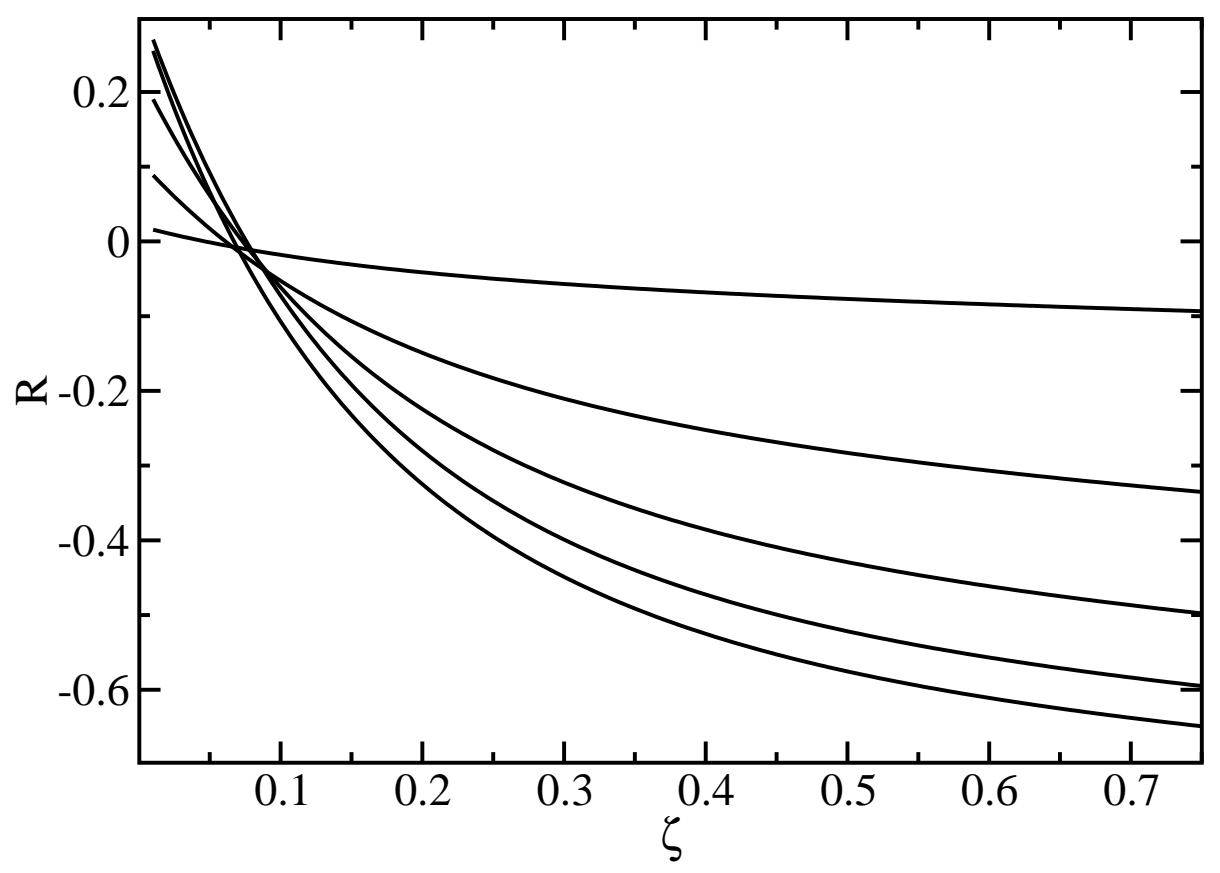

FIG. 7: The ratio $R$ in the relation (66) between the amplitudes of the number- and charge-density waves in the dominant eigenmode, along the MF spinodal lines shown in Fig.5 for $\delta=0.2$. From the top to the bottom lines (on the right) $\nu=0.9, \nu=0.5, \nu=0.0, \nu=-0.5$ and $\nu=-0.9$. Both $R$ and the volume fraction of ions $\zeta$ are dimensionless. Note that $|R|<1$, indicating periodic ordering into positively and negatively charged regions of the spacial extent $\pi / k_{b}$ (in $\sigma_{+-}$units), where $k_{b}$ is shown in Fig.6.

and

$$
\begin{array}{r}
\tilde{\eta}(\mathbf{k})=\frac{1}{\sqrt{2}}\left[\Delta \tilde{\rho}_{+}(\mathbf{k})+\Delta \tilde{\rho}_{-}(\mathbf{k})-\right. \\
\left.y\left(\Delta \tilde{\rho}_{+}(\mathbf{k})-\Delta \tilde{\rho}_{-}(\mathbf{k})\right)\right]+O\left(\delta^{2}, \delta \nu, \nu^{2}\right) \\
=\frac{1}{\sqrt{2}}[\Delta \tilde{\rho}(\mathbf{k})-y \tilde{q}(\mathbf{k})]+O\left(\delta^{2}, \delta \nu, \nu^{2}\right) .
\end{array}
$$

For infinitesimal asymmetry parameters, $\Delta \rho$ and $q$ yield infinitesimal contribution to $\phi$ and to $\eta$ respectively. The $\mathrm{OZ}$ equations are thus decoupled for the eigenmodes $\phi$ and $\eta$ that very weakly deviate from the charge- and the number densities respectively. This result agrees with the predictions of Ref. [1]. 


\section{B. Moderate and large size asymmetry}

For $\delta>0.4(\lambda>2.3)$ the structural line associated with the periodic ordering of the instantaneous states assumes a single maximum for $\zeta \approx 0.15$. Unlike the case of $\delta<0.4$, at higher volume fractions the periodic ordering is less favorable. For the nonmonotonic structural lines two cases can be distinguished, according to a different behavior of the wavenumber of the dominant instantaneous states $k_{b}$.

1. $k_{b}>\pi / 2$

Such large wavenumbers are found for the moderate size asymmetry, $0.4<\delta<0.67$, in the case of $\delta \nu>0$ (larger charge at the larger ion). For $\delta=0.6$ and $\nu>0$ the structural lines and the corresponding wavenumbers $k_{b}$ are shown in Figs.8 and 9 respectively. Except for very small $\zeta$, the wavenumber is $k_{b}>\pi / 2$, and increases with increasing $\zeta$. Increasing $k_{b}$ indicates decreasing period of oscillations of $\rho_{\alpha}(\mathbf{x})$, in agreement with expected smaller nearest-neighbor distances for larger density. For $k_{b}>\pi / 2$ the dominant field is $\phi$ (sec.IIIB). The behavior of $R$, shown in Fig.10, is similar as in the case $\delta<0.4$.

The analysis of the cluster formation in Ref.[46] was performed for this range of parameters. Namely, the case of $\delta=0.639$, and $\nu=0.85$ (monovalent), $\nu=0.7$ (divalent) and $\nu=0.6$ (trivalent counterions) was examined in detail. The volume fractions and temperatures were $\left(\zeta, T^{*}\right)=(0.0185,0.157),(0.0174,0.079)$ and $(0.017,0.0525)$ for mono-, di- and trivalent counterions respectively. All cases correspond to the low- $T^{*}$ side of the structural line, where the periodic instantaneous structures dominate. Only for trivalent counterions $k_{b}<\pi / 2$ and the relevant $\mathrm{OP}$ is $\eta$, but this fact is not sufficient to explain the differences found in the short-range ordering into clusters. Further studies beyond the stability analysis of $\Omega^{M F}$ are required to verify whether the coarse-grained description can explain the cluster formation.

\section{2. $k_{b}<\pi / 2$}

$k_{b}<\pi / 2$ is found in the case of $0.4<\delta<0.67$ for $\nu<0$ (larger charge at the smaller ion), as shown in Fig.11. 


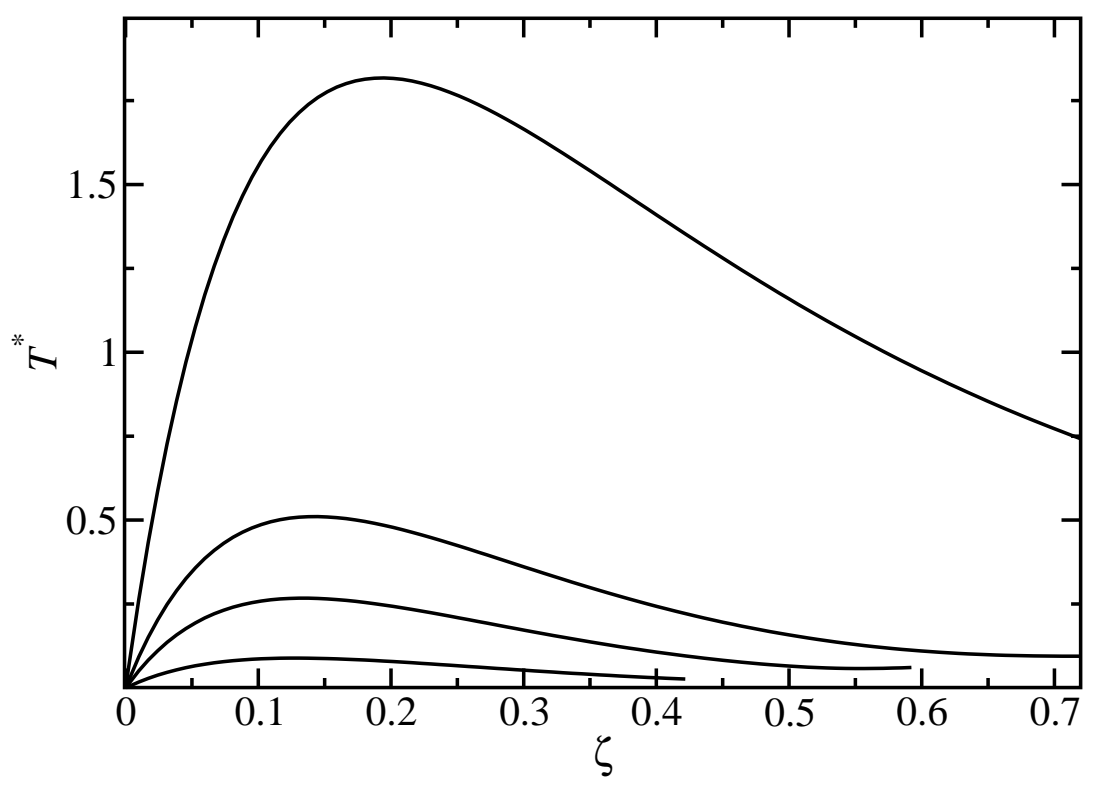

FIG. 8: MF spinodal lines of the transition to the ordered phase for $\delta=0.6$ and $\nu \geq 0$. From the top to the bottom lines $\nu=0.9, \nu=0.7, \nu=0.5$ and $\nu=0.0$. Temperature $T^{*}$ and the volume fraction of ions $\zeta$ are in dimensionless reduced units defined in Eqs.(11) and (12) respectively.

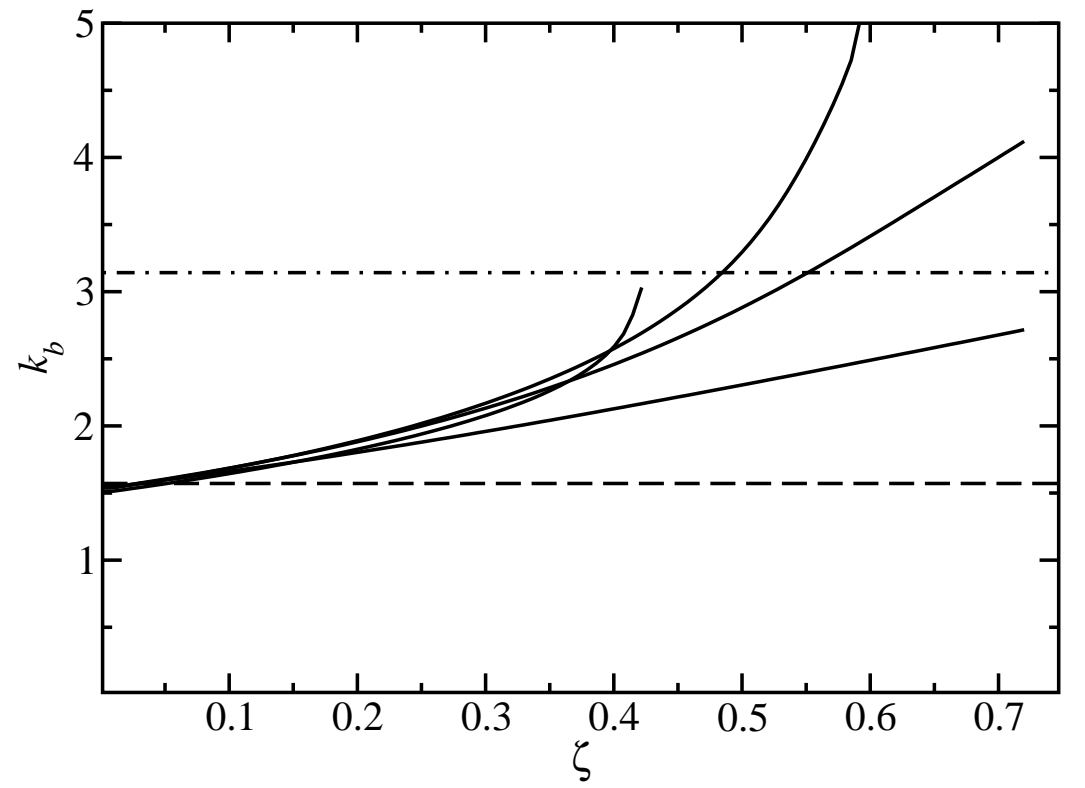

FIG. 9: The wavenumber $k_{b}$ corresponding to the ordering of ions along the MF spinodal lines shown in Fig.8 (for $\delta=0.6$ and $\nu \geq 0$ ). From the bottom to the top lines $\nu=0.9, \nu=0.7, \nu=0.5$ and $\nu=0.0 . k_{b}$ is in $\sigma_{+-}^{-1}$ units and $\zeta$ is the volume fraction of ions. The dashed and dash-dotted lines correspond to $k_{b}=\pi / 2$ and $\pi$ respectively. Note that $k_{b}<\pi / 2$ for very small values of $\zeta$. 


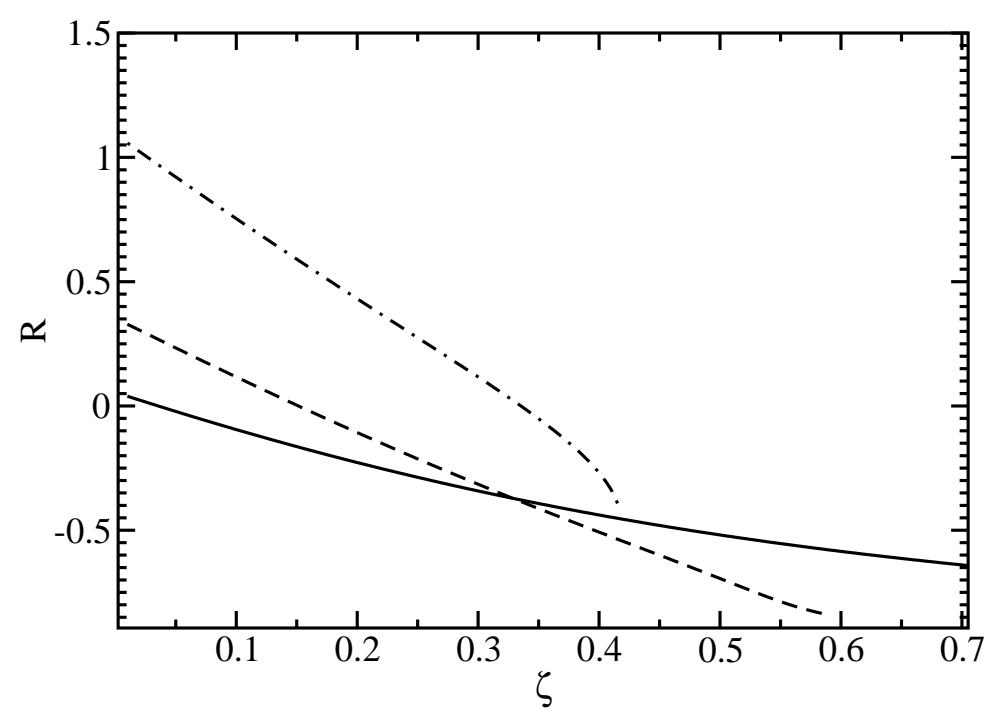

FIG. 10: The ratio $R$ (66) between the amplitudes of the number- and charge-density waves in the dominant eigenmode for $\delta=0.6$ and $\nu \geq 0$. The solid, dashed, and dash-dotted lines correspond to $\nu=0.9, \nu=0.5$ and $\nu=0.0$ respectively. Note that $|R|<1$, and periodic ordering into positively and negatively charged regions of a size $\pi / k_{b}$, with $k_{b}$ shown in Fig.9, takes place. Both $R$ and the volume fraction of ions $\zeta$ are dimensionless.

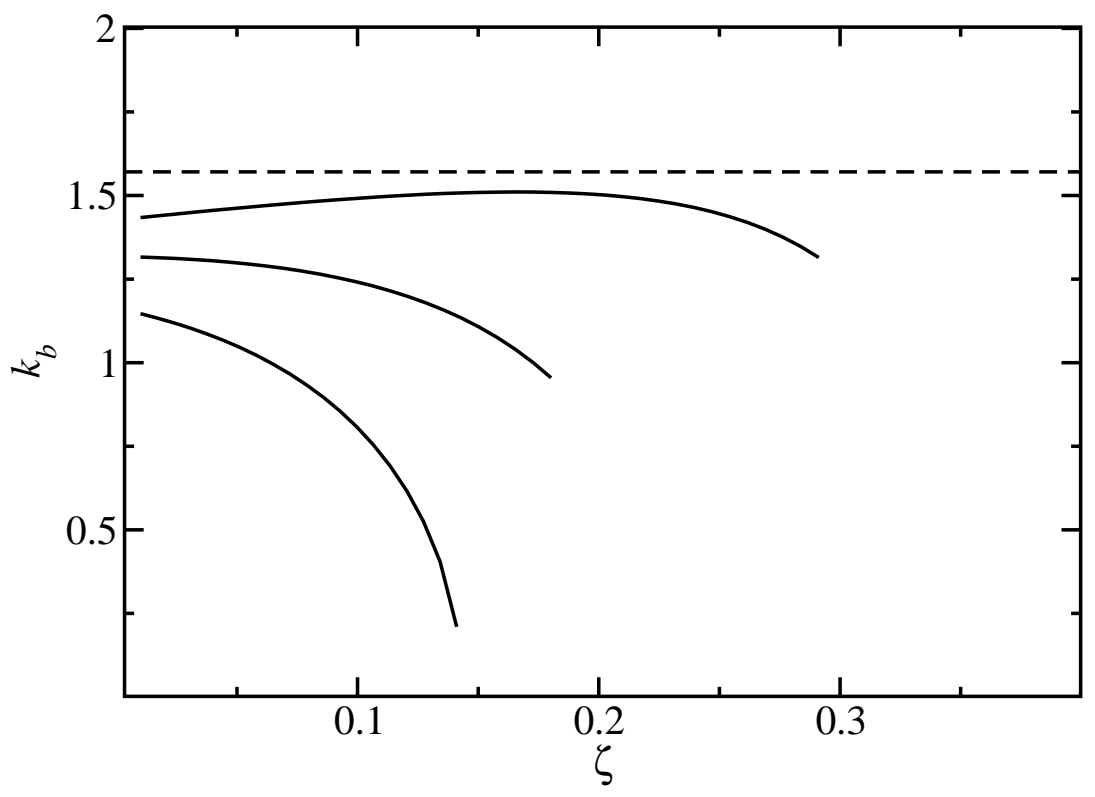

FIG. 11: The wavenumber $k_{b}$ corresponding to the ordering of ions along the MF spinodal lines shown in Fig.12 (for $\delta=0.6$ and $\nu<0$ ). From the top to the bottom lines $\nu=-0.5, \nu=-0.7$ and $\nu=-0.8 . k_{b}$ is in $\sigma_{+-}^{-1}$ units and $\zeta$ is the volume fraction of ions. At the dashed line $k_{b}=\pi / 2$. 


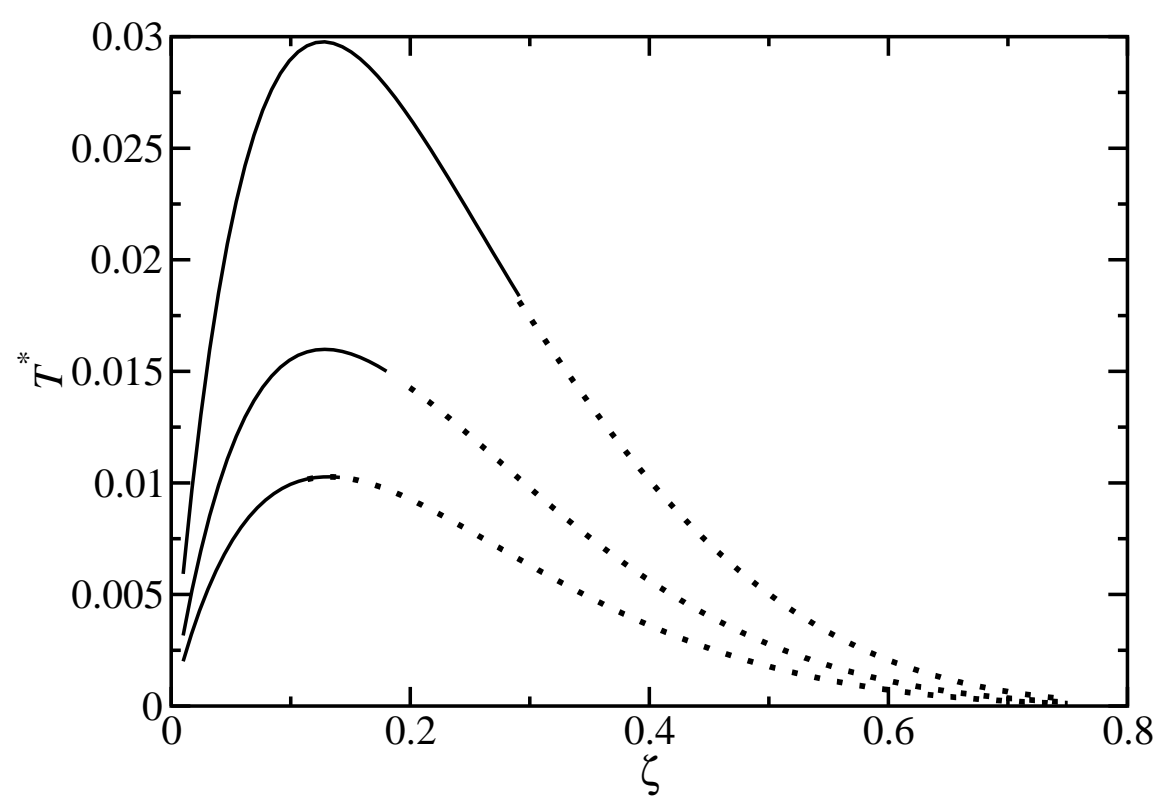

FIG. 12: Solid lines represent the MF spinodal lines of the transition to the ordered phase for $\delta=0.6$ and $\nu<0$. The dotted lines are the MF spinodals corresponding to the gas-liquid phase separation associated with $k_{b}=0$, discussed in sec.V. From the top to the bottom lines $\nu=-0.5$, $\nu=-0.7$ and $\nu=-0.8$. Temperature $T^{*}$ and the volume fraction of ions $\zeta$ are in dimensionless reduced units defined in Eqs.(11) and (12) respectively.

For $\delta>0.67(\lambda>5)$ the wavenumber of the dominant eigenmode of $\tilde{C}_{\alpha \beta}^{0}\left(k_{b}\right)$ is $k_{b}<\pi / 2$ for all values of $\nu$ (Fig.13). The extent of the phase-space region corresponding to periodic ordering of the instantaneous states is similar to that of the case of $0.4<\delta<0.67$ (Figs.14 and 15).

When the difference between $\nu$ and $\delta$ is not too large, the $k_{b}$ initially increases with increasing $\zeta$ (Fig.11 (upper curve) and 13 (two upper curves)). When $k_{b}$ increases, the structural line and the MF spinodal of the gas-liquid separations are well separated. The amplitude ratio is $R=O(1)$ and varies slowly with $\zeta$. At a value of $\zeta$ depending on $\delta$ and $\nu, k_{b}$ starts to decrease, and at some point a rapid decrease to $k_{b}=0$ occurs. At the corresponding value of $\zeta=\zeta_{L}$ (Lifshitz point) the MF spinodals merge together and become identical for $\zeta>\zeta_{L}$ (Figs.12, 14 and 15). The amplitude ratio $R$ (66) increases rapidly, when a rapid decrease to $k_{b}=0$ occurs. For a large difference between the charge and the size asymmetry, the $k_{b}$ decreases for the whole range of $\zeta$ (see the two lower curves in Fig.11 and the three lower curves in Fig. 13). 


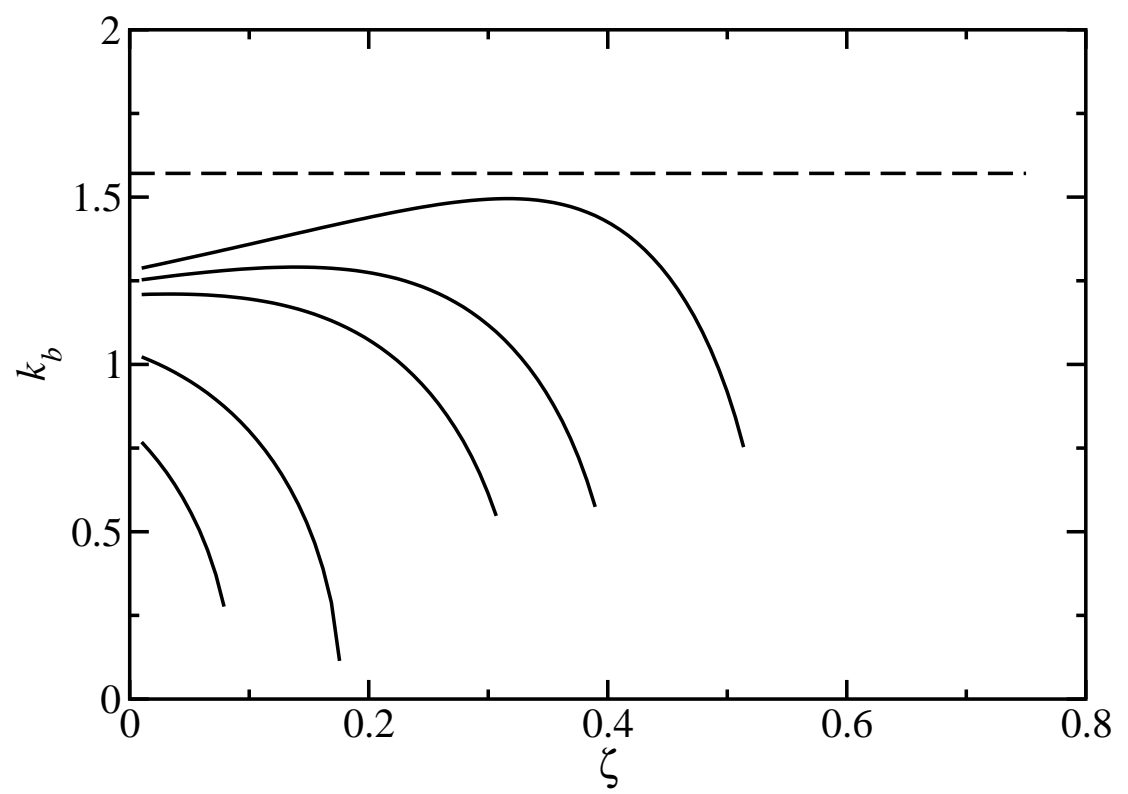

FIG. 13: The wavenumber $k_{b}$ corresponding to the ordering of ions along the MF spinodal lines for $\delta=0.9$. From the top to the bottom lines $\nu=0.9, \nu=0.7, \nu=0.5, \nu=0.0$ and $\nu=-03$. $k_{b}$ is in $\sigma_{+-}^{-1}$ units and $\zeta$ is the volume fraction of ions. The horizontal dashed line corresponds to $k_{b}=\pi / 2$.

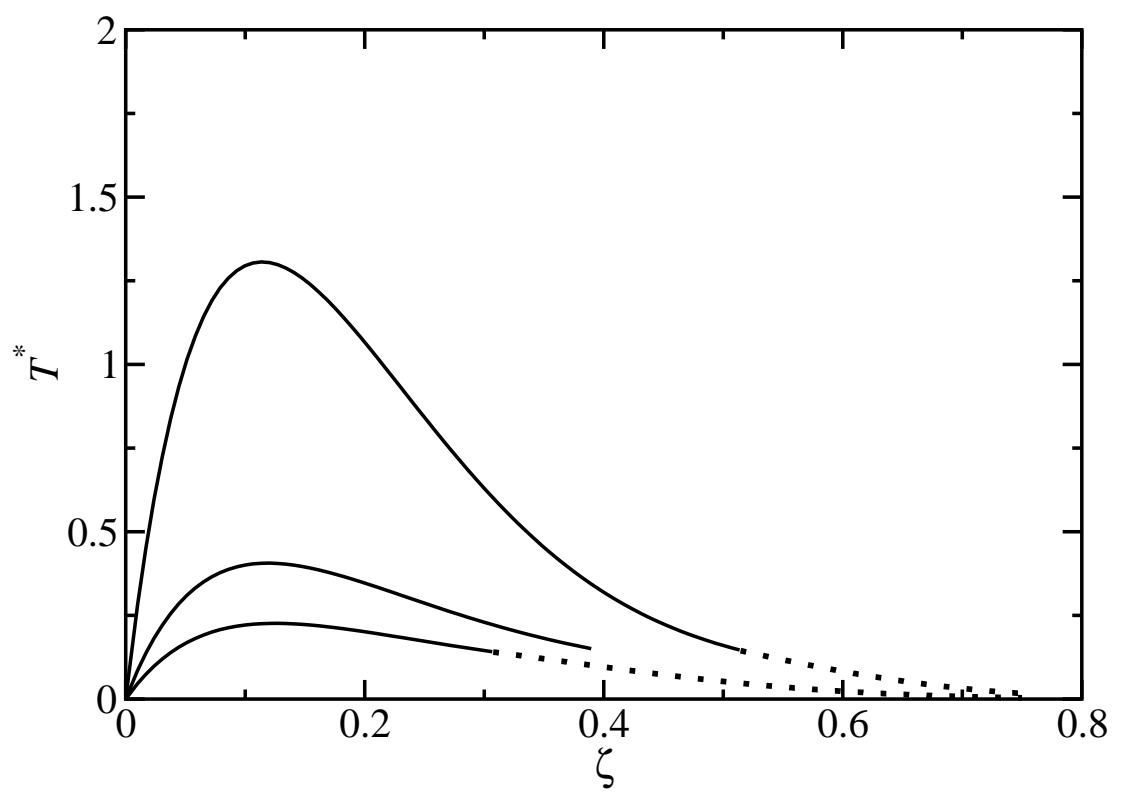

FIG. 14: MF spinodal lines (solid) for the transition to the ordered phase for $\delta=0.9$ and $\nu>0$. Dotted line represents the MF spinodal line for the gas-liquid separation. From the top to the bottom lines $\nu=0.9, \nu=0.7$ and $\nu=0.5$. Temperature $T^{*}$ and the volume fraction of ions $\zeta$ are in dimensionless reduced units defined in Eqs.(11) and (12) respectively. 


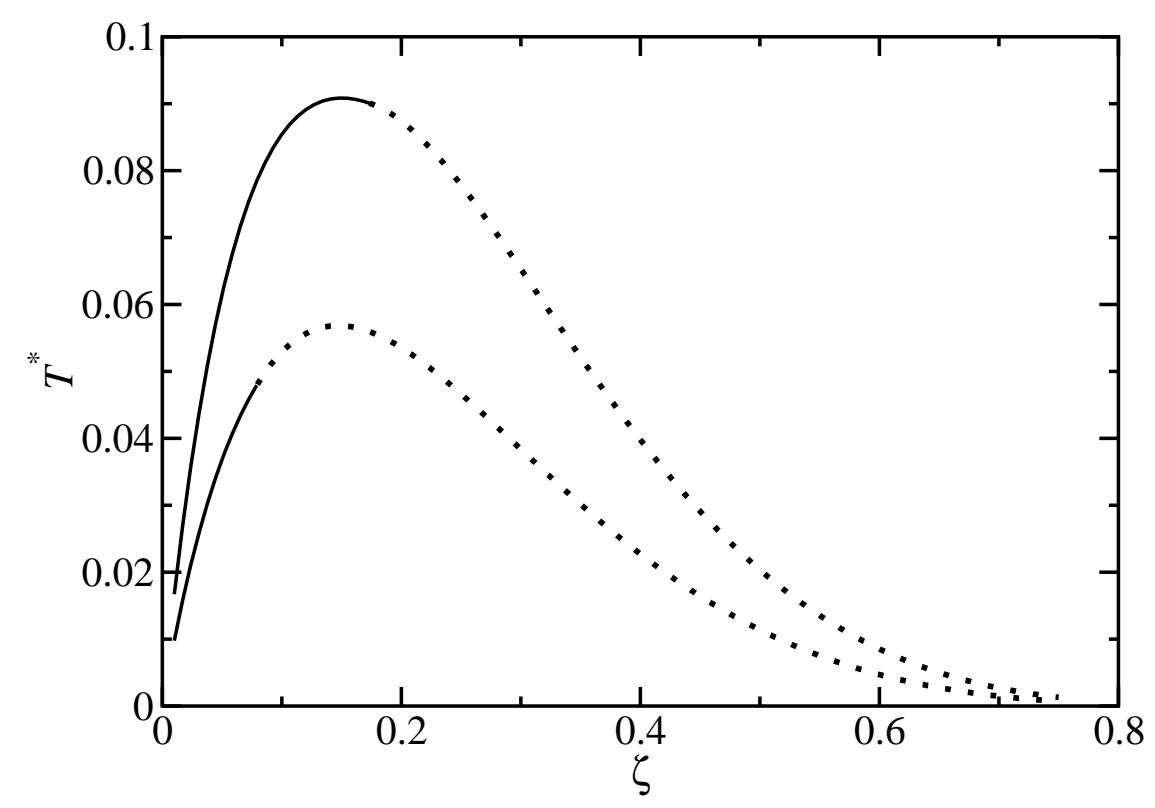

FIG. 15: MF spinodal lines (solid) for the transition to the ordered phase for $\delta=0.9$ and $\nu \leq 0$. Dotted lines are the MF spinodal lines for the gas-liquid separation. From the top to the bottom lines $\nu=0.0$ and $\nu=-0.3$. Temperature $T^{*}$ and the volume fraction of ions $\zeta$ are in dimensionless reduced units defined in Eqs.(11) and (12) respectively.

For $R \gg 1$ the amplitude of the charge-density is much smaller than the amplitude of the number-density wave (sec.IIID). Thus, the ordering into alternating oppositely charged regions is suppressed. For $\nu<0$ a large charge at the small ion is compensated by a large number of large ions having small charges; therefore the unit cell of the ordered structure is large, and may contain voids. In crystals containing voids as parts of the structure the size of the unit cell is significantly larger than the sum of radii of the two ionic species. Such voids should be distinguished from vacancies resulting from thermal fluctuations, which are present in the ionic crystals at low densities.

In this case the favorable ordered structures are much more complex than in both, the RPM-like systems and the colloid-like systems. Whether such complex structures may correspond to stable phases in the PM with moderate size- and charge asymmetry remains an open question, because the binodal associated with the periodic ordering may be preempted by the binodal associated with the phase separation.

For a large difference between the charge and the size asymmetry, the MF instability with respect to ordering of ions in periodic structures occurs only for small values of $\zeta$ and $T^{*}$ 


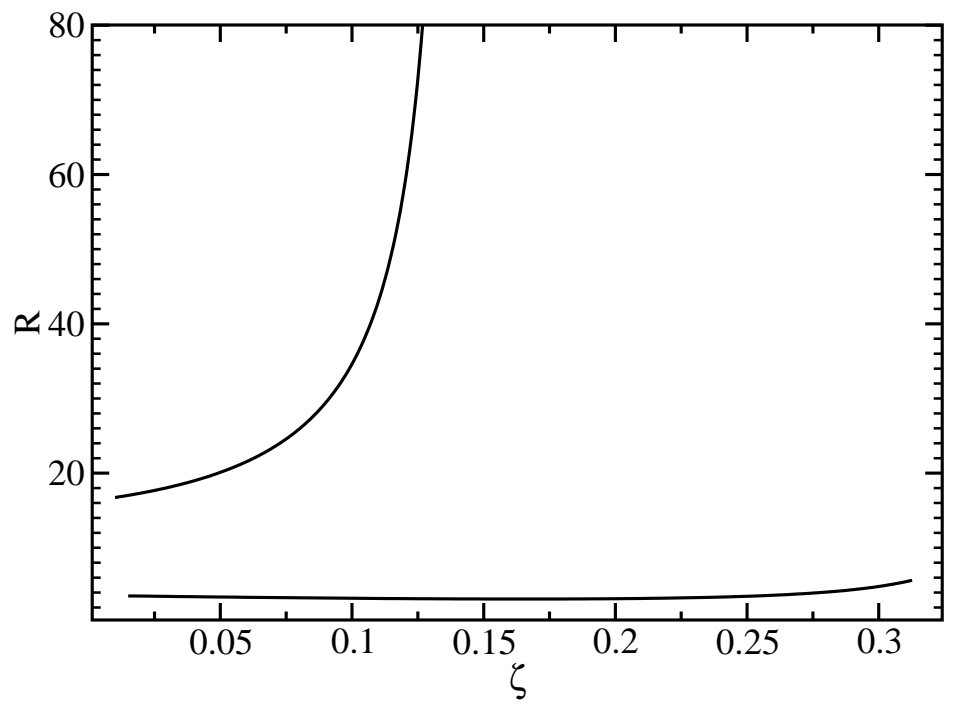

FIG. 16: The ratio $R$ (66) between the amplitudes of the number- and charge-density waves in the dominant instantaneous state for $\delta=0.6$ and $\nu<0$. The upper and the lower curve correspond to $\nu=-0.8$ and $\nu=-0.5$. Note that $R>1$, and regions of increased and depleted number density are formed. The size of these regions is $\pi / k_{b}$, with $k_{b}$ shown in Fig.11. Both $R$ and the volume fraction of ions $\zeta$ are dimensionless. In the case of the upper curve both the dense and the dilute regions in the dominant eigenmode are nearly charge neutral for $\zeta>0.1$.

(Figs. 12, 14, and 15). For $\nu \delta \rightarrow-1$ the ordering is suppressed entirely. An infinite number of large ions is required to neutralize the infinite charge at the small ion for $\nu=-1$. Clearly, formation of an ordered structure with a finite period is not possible in this (unphysical) case.

Finally, we should note that at large volume fractions the effects of ordering of hard spheres become important, and such effects are not accounted for by this theory.

\section{Case of very large asymmetry, $\delta \nu \rightarrow 1$}

The maximum of the MF spinodal $T^{*}(\zeta)$ increases when $\nu \approx \delta \rightarrow 1$, and for $\nu \approx \delta>$ 0.9 the maximum increases very rapidly. This increase of the phase-space region where a majority of the instantaneous states are the periodically ordered can be observed by comparing Figs.13, 18 and 19. In the latter plot the spinodal line and the wavevector are shown for $Z=10^{4}(\nu=0.9998)$ and $\lambda=10^{3}(\delta=0.998)$, corresponding to highly charged 


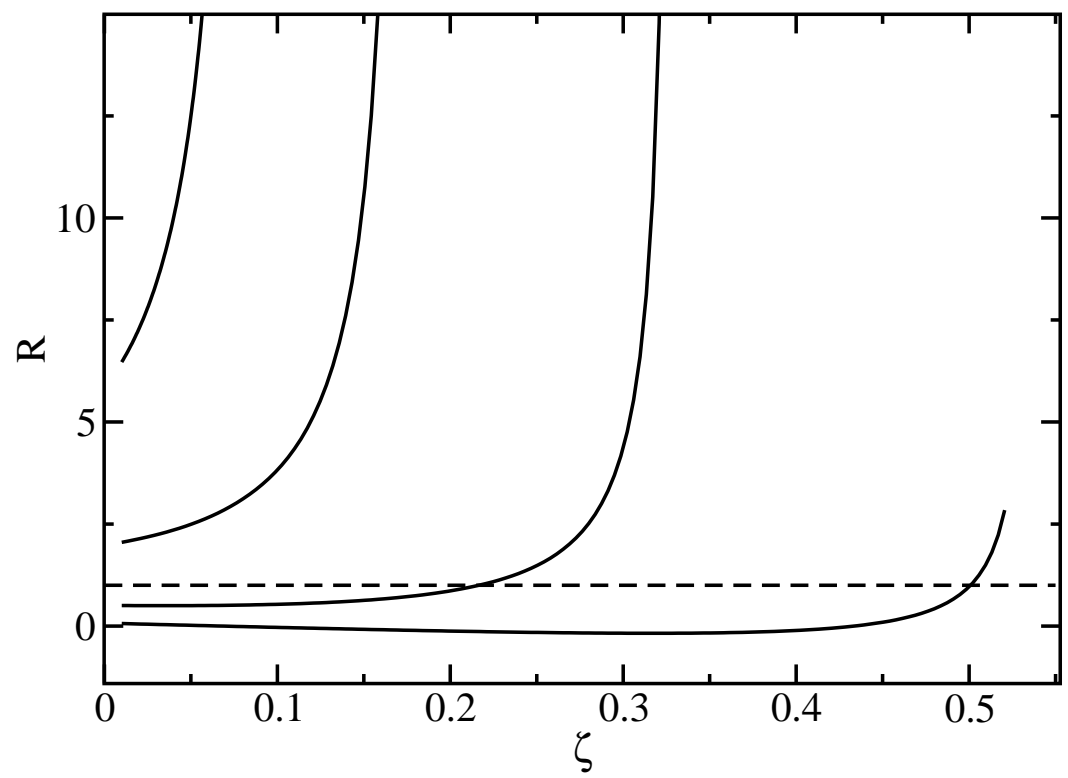

FIG. 17: The ratio $R(\underline{66})$ between the amplitudes of the number- and charge-density deviations from the average values in the dominant instantaneous state for $\delta=0.9$. From the bottom to the top lines $\nu=0.9,0.5,0$, and -0.3 . At the dashed line $R=1$. Both $R$ and the volume fraction of ions $\zeta$ are dimensionless.

colloids with counterions of a microscopic size, considered in many experimental studies.

The case of extreme asymmetry was studied in Ref. [8] for $s=O\left(Z^{0}\right)\left(\zeta=O\left(Z^{-1}\right)\right)$, i.e. for infinite dilution of colloid particles. In the limiting case the spinodal assumes the asymptotic form [8]

$$
T_{C}^{*}(\zeta)=-\frac{3 \cos \left(2 k_{C}\right)}{k_{C}^{2}} Z \zeta
$$

where

$$
\tan \left(2 k_{C}\right)=-\frac{1}{k_{C}}
$$

The subscript $C$ indicates that the MF spinodal and the corresponding wavevector correspond to the colloid limit, $\delta, \nu \rightarrow 1$, with the infinite dilution of the colloid particles for $Z \rightarrow \infty$.

Note that $k_{C}=k_{R} / 2<\pi / 2$, and according to the discussion in sec.IIIB the eigenmode $\phi$ is relevant on the high- $T^{*}$ side, whereas the eigenmode $\eta$ is relevant on the low $-T^{*}$ side of the line (52). The asymptotic analysis in Ref. [8] indicates that the phase behavior is determined only by the field $\Delta \rho_{+}$. Here we shall verify if the dominant eigenmode indeed reduces to 


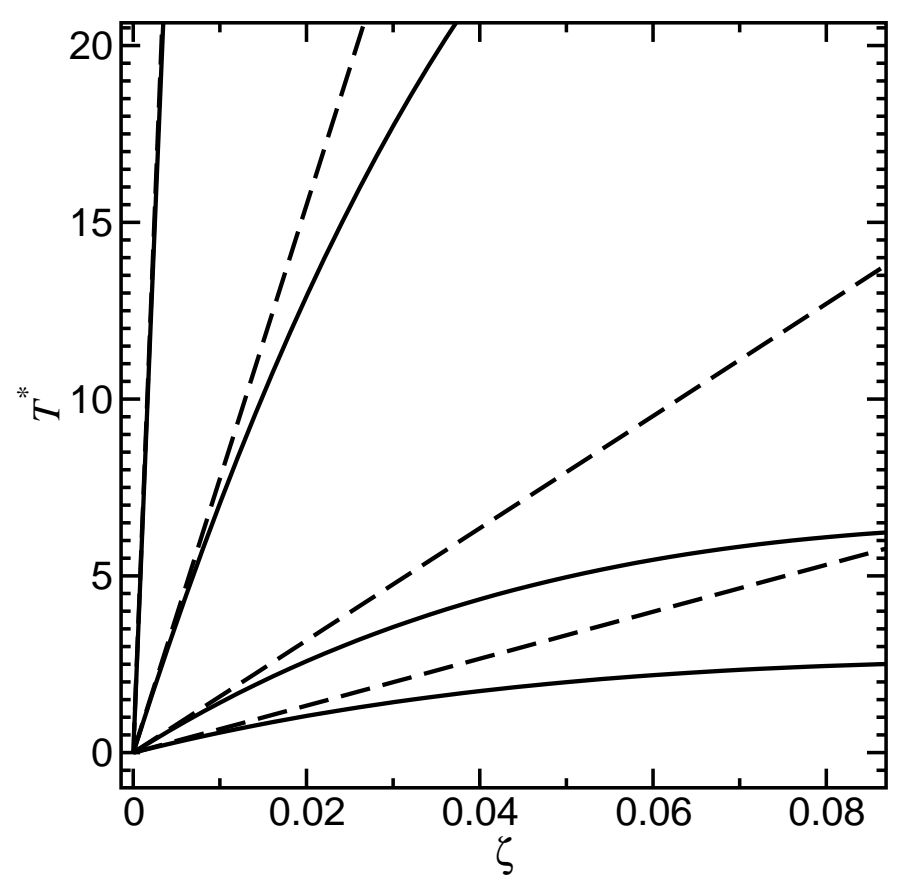

FIG. 18: The MF spinodal lines (solid) and the corresponding asymptotic behavior for $\nu=\delta \rightarrow 1$ (dashed). The latter has the form $T^{*}=12.3 \zeta /\left(1-\delta^{4}\right)$ (Eq.38 in Ref.[8] and Eq.(14) here). From the bottom to the top lines $\delta=\nu=0.95,0.98,0.996$ and $0.9995(\lambda=Z=39,99,499$ and 3999) respectively. In the last case the solid and the dashed lines are indistinguishable for the given range of $T^{*}$.

$\Delta \rho_{+}$. For $Z \rightarrow \infty$ and for $\tilde{C}_{++}(k) \neq 0$ we obtain the approximate form of Eq.(45),

$$
\mathcal{B}=\left|\tilde{C}_{++}(k)\right|-\operatorname{sign}\left(\tilde{C}_{++}(k)\right) \tilde{C}_{--}(k)+\frac{2 \tilde{C}_{+-}(k)^{2}}{\left|\tilde{C}_{++}(k)\right|}+O\left(Z^{-2}\right) .
$$

In the limit $\delta=\nu=1$ the line (52) assumes the form $T^{*}=\frac{3 \cos k}{k^{2}}$. From (49), (87) and the above we obtain the asymptotic behavior of $\tilde{a}$ and $\tilde{b}$ for $Z \rightarrow \infty$,

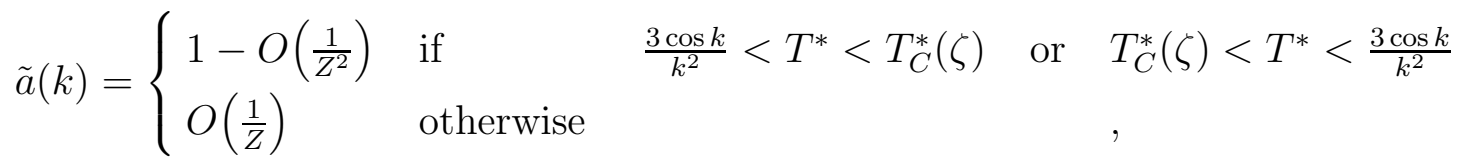

with $\tilde{b}(k)=O\left(\frac{1}{Z}\right)$ and $\tilde{b}(k)=1-O\left(\frac{1}{Z^{2}}\right)$ in the first and in the second regime respectively. The eigenmodes obtained from Eqs.(47) and (48) assume the forms

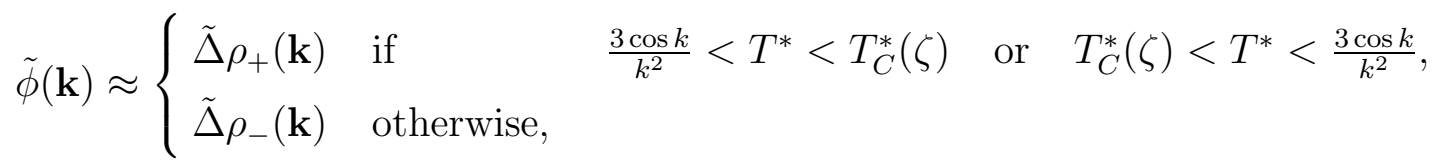



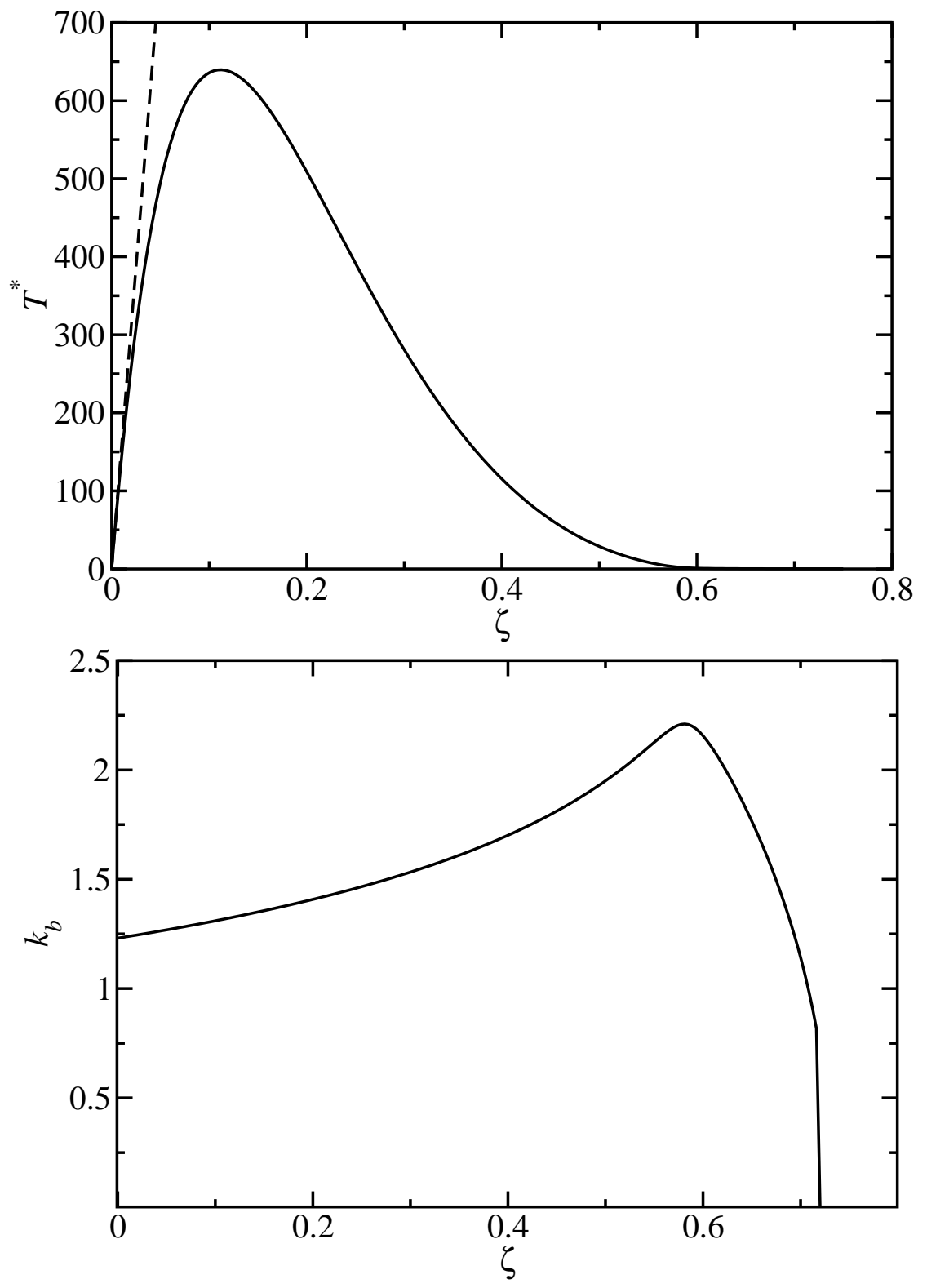

FIG. 19: The MF spinodal line (top) and the corresponding wavevector of the dominant eigenmode (bottom) for $\delta=0.998$ and $\nu=0.9998$. The dashed line is the asymptotic result of Ref. [8]. For $\zeta \ll 1, k_{b} \approx 1.23$ in agreement with the asymptotic result of Ref.[8]. $T^{*}$ is in the dimensionless units (Eq.(11)), and $k_{b}$ is in units of $\sigma_{+-}^{-1} \approx 2 / \sigma_{+}$.

and

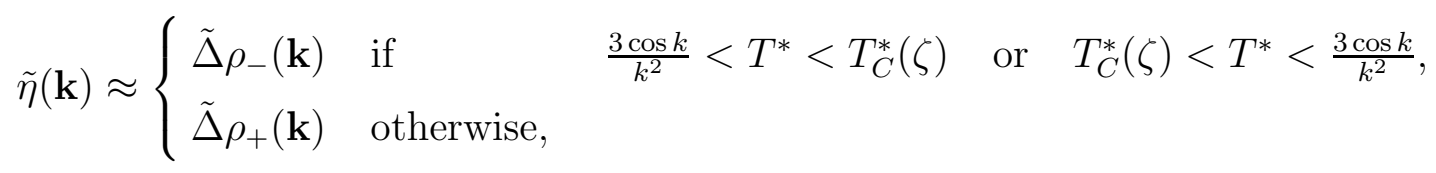

where the neglected contributions are $O(1 / Z)$. The dominant eigenmodes for different parts 


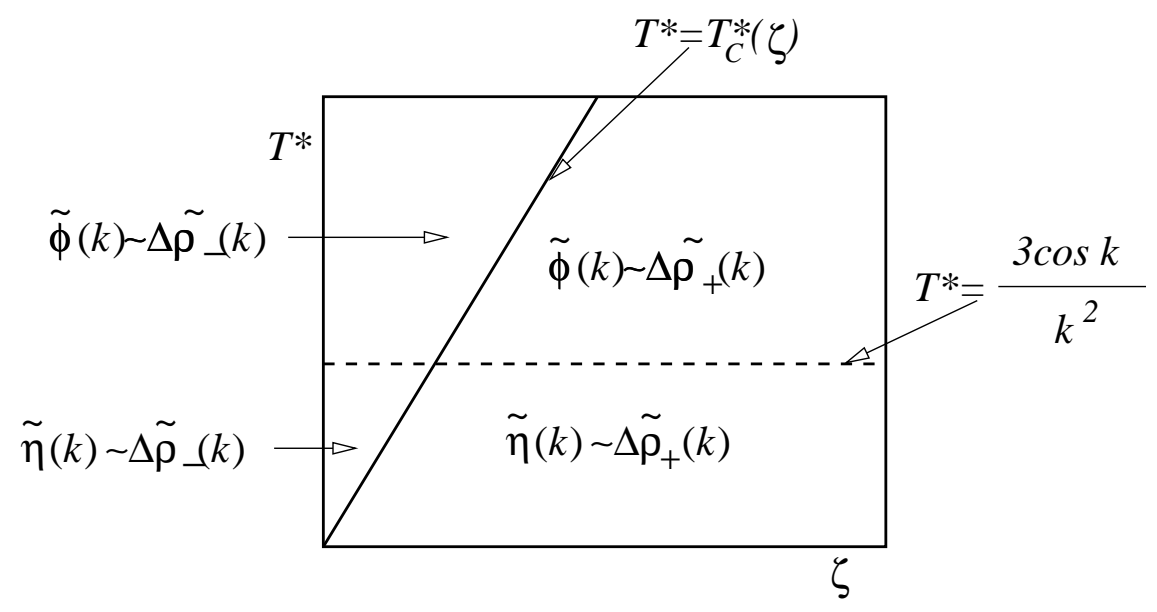

FIG. 20: Dominant eigenmodes for $\delta, \nu \rightarrow 1(\lambda, Z \rightarrow \infty)$ in different parts of the phase diagram. As discussed in sec.IIIB, the fields $\eta$ and $\phi$ dominate below and above the dashed line respectively. The relation between the eigenmodes and $\Delta \rho_{\alpha}$ is given in Eqs. (89) and (90). The neglected contributions to the OP's are $O(1 / Z)$.

of the $\left(\zeta, T^{*}\right)$ phase diagram, obtained from Eqs.(89) and (90), are shown in Fig.20. Below the MF spinodal line, i.e. for $T^{*} \leq T_{C}^{*}(\zeta)$, each dominant OP reduces to $\Delta \tilde{\rho}_{+}\left(k_{C}\right)$. The asymptotic analysis of Ref. [8] is thus fully consistent with the present, more complete approach.

\section{SUMMARY}

We performed the MF stability analysis of the disordered phase in the PM for the whole range of size and charge asymmetry of the ionic species. At the true instability the average distribution of ions undergos ordering. As we argue in sec.IID, the instabilities of the disordered phase found in MF in fact represent structural lines. The structural lines are associated with ordering of the majority of the instantaneous states, i.e. with pretransitional effects. Our results show that periodic ordering of the instantaneous states depends mainly on the size asymmetry $\delta$. Qualitative dependence on the charge asymmetry $\nu$ is found only when the size asymmetry is sufficiently large.

The strongest tendency for periodic ordering is found for $\delta, \nu \rightarrow 1$; in this case the pretransitional effects are present for a very large temperature range (Fig.19, top). The 
period of $\rho_{\alpha}(\mathbf{r}), 2 \pi / k_{b} \sim 2 \sigma_{+}$, decreases with increasing $\zeta$ (Fig.19, bottom). More detailed analysis in Ref.[8] shows that for small $\zeta$ the most probable structure has the form of the bcc crystal formed by the large ions that are immersed in the cloud of the counterions. When both asymmetry parameters decrease the temperature range for which the periodic ordering occurs decreases very rapidly (Figs.18,14,8). For $\delta, \nu=0.9, T^{*}$ at the maximum of the structural line is 500 times lower than for $\delta=0.998, \nu=0.9998$ (Figs. 14 and 19). Physical systems corresponding to this range of asymmetry parameters include highly charged colloids in the presence of one kind of counterions and no coions.

In the other extreme case of $\delta \nu \rightarrow-1$ the periodic ordering is suppressed entirely. This case is unphysical, and will not be discussed here.

For $0.9>\delta>0.4$ the periodic ordering depends on the size asymmetry rather weakly, as long as $\nu$ is sufficiently large (Figs. 8 and 14). However, when $\nu$ decreases and becomes negative (the charge at the smaller ion is not sufficiently small), $T^{*}$ and $\zeta$ corresponding to the ordering both decrease to very small values (Figs. 12 and 15). Nearly neutral clusters followed by voids are formed in this case, and the period of the structure, $2 \pi / k_{b}$, is an increasing function of $\zeta$ (Figs. 11, 13). Moreover, the corresponding structural line and the MF gas-liquid spinodal are close to each other on the phase diagram (Figs. 12 and 15). In future studies the binodal lines should be determined in MF and beyond to verify whether the complex ordered structures with large periods correspond to stable crystal phases, or whether they reflect a tendency for formation of aggregates in the fluid phase. Physical systems corresponding to this range of asymmetry may include globular proteins, organic ions, and some room-temperature ionic liquids.

For the large and moderate size asymmetry discussed above $(\delta>0.4)$ the structural line assumes a pronounced maximum for $\zeta \approx 0.15$ (Figs. $8,12,14,15,18$ ). It is remarkable that the periodic ordering induced by the Coulomb interactions is most efficient for small volume fractions, whose range, $0.1<\zeta<0.3$, is almost independent of $\delta$ (Figs. 8, 12, 14, 15, 18).

When $\delta$ further decreases, the maximum of the structural line becomes less and less pronounced, and finally dissappears for $\delta \approx 0.4(\lambda<2.3)$. $T^{*}$ at the structural line becomes a monotonically increasing function of $\zeta$, and the periodic ordering is more efficient at large volume fractions (Fig.5). The period of the ordered structure is nearly independent of $\zeta$ (sec.VIA). The same qualitative behavior is found in our MF approximation for the RPM [52]. By analogy with the RPM [37] we expect that beyond MF the ordered phase 
corresponds to a hard ionic crystal. The crystallization line is expected at much lower $T^{*}$ and much higher $\zeta$ compared to the structural line [37]. Physical systems for this range of asymmetry include molten salts and electrolytes.

Finally let us focus on the separation between two uniform phases. We found that some of the effects of the size- and charge asymmetry are correctly predicted already on the MF level of our theory (sec.V). However, on the MF level the gas-liquid separation is preempted by the instability with respect to the periodic ordering, except from the case where such ordering is suppressed by geometrical constrains. This fact agrees with earlier MF results for the RPM [41, 52, 57]. In fact, on the MF level no phase separation is found in the RPM; the phase separation is induced by the charge-charge correlations [1, 41, 52, 57, 58]. Studies beyond $\mathrm{MF}$ are required to determine for what parts of the parameter space $\left(\zeta, T^{*} ; \delta, \nu\right)$ the phase separation is indeed preempted by the crystallization, and how the critical point varies with $\delta$ and $\nu$. Returning to the clustering associated with the phase separation we should note that phenomena occurring at the very short length-scale cannot be accurately described by the coarse-grained theory. It is not clear to what extent the clustering of ions is associated with the short-distance behavior of the correlation functions, and to what extent it is induced by collective phenomena. Studies beyond the stability analysis of $\Omega^{M F}$ may shed light on this question.

Short-range interactions of different origin (including solvet-induced effective interactions between solute molecules) also play an important role for the phase behavior [3]. Such short-range interactions can be included in the field-theoretic approach by supplementing the energy contribution to $\Omega^{M F}$ in Eq.(7) with additional terms.

\section{Acknowledgments}

GS gratefully acknowledges the support of the Division of Chemical Sciences, Office of the Basic Energy Sciences, Office of Energy Research, US Department of Energy. The work of AC and WTG was partially funded by the KBN grant No. 1 P03B 03326. 


\section{APPENDIX A}

The quantities $a_{\alpha \beta}$ in Eq.(20) are obtained from the explicit expressions for the chemical

potentials in the hard-sphere mixture derived in Ref. [48]. Direct differentiations lead to the following expressions

$$
a_{++}=\frac{1}{\rho_{0+}^{*}}+\frac{\pi r_{+}^{3}}{6}\left[\frac{8}{1-\zeta}+\frac{6 r_{+}^{2} Y+15 r_{+} X+r_{+}^{3} s}{(1-\zeta)^{2}}+\frac{18 r_{+}^{2} X^{2}+6 r_{+}^{3} X Y}{(1-\zeta)^{3}}+\frac{9 r_{+}^{3} X^{3}}{(1-\zeta)^{4}}\right]
$$

with the analogous expression for $a_{--}$,

$$
a_{+-}=\frac{\pi}{6}\left[\frac{8}{1-\zeta}+\frac{2 r_{+} r_{-}\left(6+r_{+} r_{-}\right) X+8 r_{+}^{2} r_{-}^{2} Y}{(1-\zeta)^{2}}+\frac{18 r_{+}^{2} r_{-}^{2} X^{2}+6 r_{+}^{3} r_{-}^{3} X Y}{(1-\zeta)^{3}}+\frac{9 r_{+}^{3} r_{-}^{3} X^{3}}{(1-\zeta)^{4}}\right.
$$

where $s, \zeta$ and $r_{ \pm}$are defined in Eqs. (13), (12) and (24), and following Ref. [48] we have introduced the notation

$$
\begin{gathered}
X=\frac{\pi}{6}\left(r_{+}^{2} \rho_{0+}^{*}+r_{-}^{2} \rho_{0-}^{*}\right)=s\left(1+\delta^{2}-2 \delta \nu\right) \\
Y=\frac{\pi}{6}\left(r_{+} \rho_{0+}^{*}+r_{-} \rho_{0-}^{*}\right)=s(1-\delta \nu) .
\end{gathered}
$$

\section{APPENDIX B}

The functions $b$ and $d$ introduced in Eq. (57) are defined by

$$
\begin{gathered}
b=\left(a_{++} Z^{-1}+a_{--} Z\right) \cos k \cos (k \delta)+\left(a_{++} Z^{-1}-a_{--} Z\right) \sin k \sin (k \delta)+2 a_{+-} \cos k, \\
d=a_{++} a_{--}-a_{+-}^{2}=\frac{1}{\rho_{0+}^{*} \rho_{0-}^{*}} \frac{(1+2 \zeta)^{2}}{(1-\zeta)^{4}}>0
\end{gathered}
$$

[1] G. Stell, J. Stat. Phys. 78, 197 (1995).

[2] M. E. Fisher, J. Stat. Phys. 75, 1 (1994).

[3] A. Ciach and G. Stell, Int.J. Mod. Phys. B 19, 3309 (2005).

[4] T. Zemb et al., Curr. Opinion Colloid Int. Sci 9, 74 (2004).

[5] F. W. Tavares, D. Bratko, and J. M. Prausnitz, Curr. Opinion Colloid Int. Sci 9, 81 (2004).

[6] Y. Levin, Rep. Prog. Phys. 65, 1577 (2002).

[7] C. Vega, J. L. F. Abascal, C. McBride, and F. Bresme, J. Chem. Phys. 119, 964 (2003). 
[8] A. Ciach, W. T. Góźdź, and G. Stell, J. Phys. Condens. Mat. 18, 1629 (2006).

[9] Y. V. Kalyuzhnyi, M. F. Holovko, and V. Vlachy, J. Stat. Phys. 100, 243 (2000).

[10] D. M. Zukerman, M. E. Fisher, and S. Bekiranov, Phys. Rev. E 64, 11206 (2001).

[11] M. N. Artyomov, V. Kobelev, and A. B. Kolomeisky, J. Chem. Phys. 118, 6394 (2003).

[12] J.-N. Aqua and M. E. Fisher, Phys. Rev. Lett. 92, 135702 (2004).

[13] F. O. Raineri, J. P. Routh, and G. Stell, J. Phys. IV (France) 10, 99 (2000).

[14] Y. Qin and J. M. Prausnitz, J. Chem. Phys. 121, 3181 (2004).

[15] J.-N. Aqua, S. Banerjee, and M. E. Fisher, Phys. Rev. E 72, 041501 (2005).

[16] W. Zhou and J. Percus, Phys. Rev. Lett. 95, 235701 (2005).

[17] O. Patsahan, I. Mryglod, and T. Patsahan, (2006), e-print cond-mat/0606687.

[18] J. M. Romero-Enrique, G. Orkoulas, A. Panagiotopoulos, and M. Fisher, Phys. Rev. Lett. 85, 4558 (2000).

[19] Q. Yan and J. de Pablo, Phys. Rev. Lett. 86, 2054 (2001).

[20] Q. Yan and J. de Pablo, Phys. Rev. Lett. 88, 95504 (2002).

[21] A. Panagiotopoulos and M. Fisher, Phys. Rev. Lett. 88, 45701 (2002).

[22] D. Cheong and A. Panagiotopoulos, J. Chem. Phys. 119, 8526 (2003).

[23] J. Rescic and P. Linse, J. Chem. Phys. 114, 10131 (2001).

[24] P. Linse, Philos. Trans. R. Soc. London, Ser.A 359, 853 (2001).

[25] B. Derjaguin and L. D. Landau, Acta Physicochim. URSS 14, 633 (1941).

[26] E. J. W. Verwey and J. T. G. Overbeek, Theory of the Stability of Lyophobic Collids (Elsevier, Amsterdam, 1948).

[27] P. B. Warren, J. Chem. Phys. 112, 4683 (2000).

[28] R. van Roij, M. Dijkstra, and J.-P. Hansen, Phys. Rev. E 59, 2010 (1999).

[29] N. Ise et al., J. Chem. Phys. 78, 536 (1983).

[30] N. Ise, T. Konish, and B. Tata, Langmuir 15, 4176 (1999).

[31] K. Ito, H. Yoshida, and N. Ise, Science 263, 66 (1994).

[32] L. Belloni and O. Spalla, J. Chem. Phys. 107, 465 (1997).

[33] L. Belloni, J. Phys.:Cond. Mat. 12, R549 (2000).

[34] B. V. R. Tata, E. Yamahara, P. V. Rajamani, and E. Ise, Phys. Rev. Lett. 78, 2660 (1997).

[35] R. Kjellander and D. J. Mitchell, Mol. Phys. 91, 173 (1997).

[36] A. K. Arora and B. V. R. Tata, Adv. Colloid Interface Sci. 78, 49 (1998). 
[37] A. Ciach and O. Patsahan, Phys. Rev. E 74, 021508 (2006).

[38] N. Choudhury and S. K. Ghosh, Phys. Rev. E 51, 4503 (1995).

[39] H. Löwen, Phys. Rep. 237, 249 (1994).

[40] Y.-X. Yu, J. Wu, and G.-H. Gao, J. Chem. Phys. 120, 7223 (2004).

[41] A. Ciach, Phys. Rev. E 73, 066110 (2006).

[42] B. Hribar and V. Vlachy, J. Phys. Chem B. 101, 3457 (1997).

[43] B. Hribar and V. Vlachy, Biophys. J. 78, 694 (2000).

[44] Q. Yan and J. J. de Pablo, J. Chem. Phys. 116, 2967 (2002).

[45] J.-J. Weis, D. Levesque, and J. Caillol, J. Chem. Phys. 109, 7486 (1998).

[46] E. Spohr, B. Hribar, and V. Vlachy, J. Phys. Chem B. 106, 2343 (2002).

[47] M. Jardat, T. Cartailler, and P. Turq, J. Chem. Phys. 115, 1066 (2001).

[48] J. L. Lebowitz and J. S. Rowlinson, J. Chem. Phys. 41, 133 (1964).

[49] O. Patsahan and I. Mryglod, J. Phy.:Condens. Mat. 39, L583 (2006).

[50] I. R. Yukhnovskii, Sov.Phys. JETP 34, 263 (1958).

[51] I. R. Yukhnovskii, Phase Transitions of the Second Order, Collective Variable Methods (World Scientific, Singapore, 1978).

[52] A. Ciach and G. Stell, J. Mol. Liq. 87, 255 (2000).

[53] R. Evans, Advances in Phys. 28, 143 (1979).

[54] Zinn-Justin, Quantum Field Theory and Critical Phenomena (Clarendon Press, Oxford, 1989).

[55] A. Ciach, Phys. Rev. E 70, 046103 (2004).

[56] S. A. Brazovskii, Sov. Phys. JETP 41, 8 (1975).

[57] A. Ciach and G. Stell, J. Chem. Phys. 114, 382 (2001).

[58] O. V. Patsahan and I. M. Mryglod, Cond.Matt.Phys. 7, 755 (2004).

[59] R. R. Netz and H. Orland, Europhys. Lett. 45, 726 (1999).

[60] J. M. Caillol, Mol. Phys. 103, 1271 (2005).

[61] J. M. Caillol, J. Stat. Phys. 115, 1461 (2004). 